Accepted for publication in ApJ

Preprint typeset using $\mathrm{L}_{\mathrm{A}} \mathrm{T}_{\mathrm{E}} \mathrm{X}$ style emulateapj v. 6/22/04

\title{
CHARACTERIZING THE NEAR-UV ENVIRONMENT OF M DWARFS
}

\author{
Lucianne M. Walkowicz ${ }^{1}$, Christopher M. Johns-Krull ${ }^{2}$, Suzanne L. Hawley ${ }^{1}$ \\ Accepted for publication in ApJ
}

\begin{abstract}
We report the results of our HST snapshot survey with the ACS HRC PR200L prism, designed to measure the near-UV emission in a sample of nearby $\mathrm{M} \mathrm{dwarfs}^{1}$. 33 stars were observed, spanning the mass range from $0.1-0.6$ solar masses $\left(\mathrm{T}_{\text {eff }} \sim 2200 \mathrm{~K}-4000 \mathrm{~K}\right)$ where the UV energy distributions vary widely between active and inactive stars. These observations provide much-needed constraints on models of the habitability zone and the atmospheres of possible terrestrial planets orbiting M dwarf hosts, and will be useful in refining the target selection for future space missions such as TPF. We compare our data with a new generation of $\mathrm{M}$ dwarf atmospheric models and discuss their implication for the chromospheric energy budget. These NUV data will also be valuable in conjunction with existing optical, FUV and X-ray data to explore unanswered questions regarding the dynamo generation and magnetic heating in low-mass stars.
\end{abstract}

Subject headings: stars: low mass — stars: magnetic activity — stars: emission lines — stars: flares

\section{INTRODUCTION}

M dwarfs are by far the most numerous of nearby stars, comprising roughly $75 \%$ of the Galactic stellar population. The sheer number of $\mathrm{M}$ dwarfs in our local solar neighborhood makes them attractive targets for planetfinding missions, while their low masses make them a likely place for discovering Earth-mass worlds in radial velocity surveys. However, these cool neighbors also pose a unique set of problems for their resident planets, and so have been largely dismissed in years past in favor of more tractable cases.

Recently, however, there has been renewed interest in M dwarfs as hosts for habitable worlds. The case for M dwarf planets has been outlined in Tarter et al. (2007), bringing together studies by Joshi et al. (1997), Joshi (2003), Segura et al. (2005) and others to propose that perhaps the case for habitability is not so dire after all. As further timely motivation, Udry et al. (2007) recently announced the discovery of the lowest mass planet to date $\left(\mathrm{M}_{p} \sim 5 \mathrm{M}_{\oplus}\right)$, orbiting in the habitable zone of a M3.5 dwarf, Gl 581.

The near-UV emission of $M$ dwarfs remains an outstanding unknown, affecting both our understanding of the basic atmospheric structure in these stars and the potential habitability of attendant planets. Due to their cool effective temperatures, $\mathrm{M}$ dwarfs exhibit almost no continuum emission in the near-UV. However, a large fraction of these stars possess surface magnetic fields, which give rise to a steady-state, hot outer atmosphere and transient events such as starspots and flares. Nonradiative heating processes transfer energy from the magnetic field into the stellar atmosphere to power these phenomena, producing significant UV output in the form of

\footnotetext{
1 Astronomy Department, University of Washington, Box 351580, Seattle, WA 98195

2 Department of Physics and Astronomy, Rice University, 6100 Main Street, Houston, TX 77005

${ }^{1}$ Based on observations made with the NASA/ESA Hubble Space Telescope, obtained at the Space Telescope Science Institute, which is operated by the Association of Universities for Research in Astronomy, Inc., under NASA contract NAS 5-26555. These observations are associated with program 10525.
}

chromospheric $\left(\mathrm{T} \sim 10^{4} \mathrm{~K}\right)$, transition region $\left(\mathrm{T} \sim 10^{5} \mathrm{~K}\right)$ and coronal $\left(\mathrm{T} \sim 10^{6} \mathrm{~K}\right)$ emission. Chromospheric nearUV emission can have critical consequences for planetary atmospheres, as it directly impacts the habitability of a neighboring planet through its role in the production of ozone, as well as its implications for long-term survival (and thus detectability) of biomarker gases (such as $\mathrm{N}_{2} \mathrm{O}$ and $\mathrm{CH}_{4}$ ). Preliminary studies of $\mathrm{M}$ dwarf habitability zones indicate that the typical chromospheric UV flux from an active star will produce observable ozone layers, comparable to that of Earth, on terrestrial planets around M dwarfs (see Segura et al. (2005), Figure 3). However, models of planetary atmospheres around relatively inactive $\mathrm{M}$ stars are presently based on stellar model photosphere predictions due to the lack of nearUV observational data.

The data described in this paper also provide crucial constraints for new model atmospheres, which may be used to address a number of outstanding problems in stellar astrophysics. For example, optical $(\mathrm{H} \alpha)$ observations of nearby stars (Joy \& Abt (1974); Reid et al. (1995); Hawley et al. (1996); Gizis et al. (2000); West et al. (2004)) show that the active fraction (as measured by $\mathrm{H} \alpha$ emission with equivalent width $>1 \AA$ ) increases dramatically from types M0 to M6, peaks near spectral type M7-M8 and declines thereafter. The strength of activity, as measured by the ratio of the luminosity in $\mathrm{H} \alpha$ compared to the bolometric luminosity $\left(L_{H \alpha} / L_{b o l}\right)$, also declines at late types (Burgasser et al. 2002; Cruz \& Reid 2002; West et al. 2004). In addition, it has been suggested that activity in ultracool dwarfs is confined primarily to large flares, with little or no "quiescent" emission (Rutledge et al. (2000); Linsky et al. (1995); Fleming, Giampapa, \& Schmitt (2000)). In contrast, STIS/MAMA observations of active M7-M9 dwarfs by Hawley \& Johns-Krull (2003) showed that quiescent transition region (C IV) emission is present at levels comparable to those seen in earlier active $\mathrm{M}$ dwarfs.

The difficulty in interpreting these intriguing empirical data lies primarily in our lack of a theory of magnetic dynamo field production and subsequent atmospheric heating. At types later than about M3, stars 
become fully convective and a solar-type shell dynamo can no longer operate. The theory of $\alpha^{2}$ or turbulent dynamos is not yet developed enough to provide predictions or guidance regarding the efficacy of magnetic field production. Progress may be made, however, on the atmospheric heating problem. It has not been established whether the drop in active fraction and activity level seen at types later than M7 is a result of the particular diagnostic we are using $(\mathrm{H} \alpha)$. If magnetic heating is still present, but the atmospheric structure has changed in these late type stars, emission may occur preferentially in other lines. In particular, the relatively unobserved 1700-3000^ wavelength region contains numerous strong emission lines important to the energy balance of the outer atmosphere, including the resonance lines of $\mathrm{Mg}$ II and a host of Fe II emission lines. These lines provide additional information on the atmospheric structure at chromospheric and transition region temperatures, and therefore to the underlying heating processes.

\section{OBSERVATIONS AND ANALYSIS}

Our sample comprises 107 nearby low mass dwarfs, extensively studied in the Palomar/Michigan State University (PMSU) survey of nearby stars [Reid et al. (1995), Hawley et al. (1996)]. We have homogeneous optical spectra for nearly all of these stars, which allowed us to choose a wide distribution of spectral types and chromospheric activity strengths (the equivalent width of the $\mathrm{H} \alpha$ line is used a proxy for chromospheric activity). Prior spectroscopic analysis of these stars also allowed us to select our sample to exclude known spectroscopic binaries. Roughly half of the stars in our sample were previously observed with the International Ultraviolet Explorer (IUE) at near-UV wavelengths (see discussion, Section 3.1). In addition, HST/STIS spectra of the NUV and transition region FUV emission are available for several of the most active stars in our sample.

The Hubble Space Telescope (HST) observed $29 \mathrm{M}$ dwarfs and 2 late $\mathrm{K}$ dwarfs from our sample using the Advanced Camera for Surveys High Resolution Channel (ACS HRC) with the PR200L prism in Snapshot mode. Table 1 provides a summary of the observed sample. Observations spanned the period of 3 August 2005 to 11 June 2006, terminating with an electronics glitch in the HRC and subsequent safing of the instrument. The observed sample ranges from spectral type K7 to M5 and includes both inactive and active stars. All but two of the observed stars lie within 10 parsecs of the Sun.

With the failure of the STIS instrument in 2004, the PR200L prism became the only spectroscopic element on board HST capable of near-UV spectroscopic obsevations. The PR200L has a highly nonlinear dispersion, ranging from $5.3 \AA \mathrm{pix}^{-1}$ at $1800 \AA$ to $105 \AA$ pix $^{-1}$ at $3500 \AA$. By $5000 \AA$ the dispersion decreases to $563 \AA$ $\mathrm{pix}^{-1}$, in essence depositing all redwards flux onto just a few pixels of the CCD detector. The rapidly decreasing dispersion towards longer wavelengths results in what has become known as the "red pile-up" 3 , making the PR200L data problematic for the study of intrinsically red objects, where scattered light from the red end can contaminate or drown the faint UV signal. Fortunately, diffraction spikes from the red pileup lie at an angle to the

\footnotetext{
${ }^{3}$ HST Instrument Science Report 0603
}

dispersion direction, causing the degree of contamination to lessen towards shorter wavelengths. Taking advantage of this fact, we were able to choose an extraction window that minimized the scattered light contamination.

Spectra were extracted in PyRAF using the aXe slitless spectroscopy reduction package, which consists of several tasks designed to handle large format spectroscopic slitless images. Observations for an individual star consist of a "direct" or undispersed image taken with the F330W filter, followed by a dispersed image taken with the PR200L prism. In the reductions, the position of the star on the direct image is used in combination with telescope position information from the header to locate the spectrum on the subsequent dispersed image and assign a wavelength to each pixel in the spectrum. aXe first generates pixel extraction tables (PETs), which contain a spectral description for each pixel in the spectrum. Pixels in the extraction window are then projected onto the spectral trace in individual wavelength bins, and their signal is weighted by the fractional area of the pixel that falls in a given bin. Flux calibration follows using sensitivity curves for the relevant observing mode (here, the HRC with PR200L prism). Although the potential for contamination by neighboring objects in an image exists for all slitless spectroscopy (e.g. when dispersing stars that lie in a crowded field), all of our targets were either solitary or well separated from others in their images, so geometric contamination was not an issue.

The reduction tasks require very little user interaction, most information being supplied by STSDAS configuration files or drawn from the image headers themselves. At the user's discretion, however, are the parameters of the extraction window. As the diffraction spikes from the red pileup lie very close to the NUV spectrum, a careful balance must be struck between including as much of the already-meager NUV flux as possible, while excluding the influence of the nearby artifact. As previously mentioned, the diffraction spikes lie at an angle to the spectrum, merging with the spectrum at the red end. The artifact and spectrum diverge towards shorter wavelengths, but if a wide extraction window is used, part of the artifact may still be included. The wavelength extent of contamination is therefore a function of the width of the extraction window, where a wide window includes the artifact over more of the spectrum than a narrow window does. In order to quantify the effect of the artifact on the output spectrum, we experimented both with a variety of extraction windows centered on the spectrum, and with extractions offset below the spectrum (i.e. a slice across the diffraction spike) and above the spectrum (where no artifact was present). After much experimentation, we determined a narrow extraction window half-width of 1 pixel to be the best choice. To quantify the uncertainty in our spectra, we extracted a spectrum from the background (offset 3 pixels above the spectrum) to examine the pixel-to-pixel variation. Variations in the background spectra were used to compute the error bars that are shown on all plots in this paper.

\section{RESULTS}

Figure 1 shows the resulting near-UV spectrum for one of the most active stars in our sample, AD Leo (dM3e, $\mathrm{d}=4.9 \mathrm{pc}$ ). Note that the uncertainty in the flux increases towards longer wavelengths as contamination from the 
red pileup becomes more apparent. In Figure 1 we also compare prior IUE observations of AD Leo to our extracted ACS spectrum. Plotted at top is the IUE spectrum, taken with the low resolution grating. At center we plot a simulated prism spectrum generated by using the IUE spectrum as input to SLIM, the ACS slitless spectroscopy simulator ${ }^{4}$. SLIM uses an input spectrum of arbitrary resolution to simulate an ACS grism or prism image using a specified configuration (in our case, the HRC with the PR200L prism). As SLIM does not account for the artifacts introduced by the red pileup, the simulated spectrum represents a "best-case scenario", devoid of contaminating scattered light. The simulated spectrum shows three main features: a peak at $2800 \AA$, attributable to the strong $\mathrm{Mg}$ II $\mathrm{h}$ and $\mathrm{k}$ lines, and two other peaks, at $2600 \AA$ and $2400 \AA$, both due to collections of strong Fe II lines clustered around those wavelengths. These features are also seen in the observed ACS spectrum for $\mathrm{AD}$ Leo, plotted at the bottom of the figure. The flux in the $\mathrm{Mg}$ II feature at $2800 \AA$ matches almost exactly between the simulated and observed spectra, and the fluxes in the two Fe II features at $2600 \AA$ and $2400 \AA$ are also quite comparable.

Figure 2 plots the observed NUV spectra for the entire sample, in approximate order of strong to weak ultraviolet emission. For the most active stars, we clearly resolve the main spectral features discussed in Figure 1. As activity decreases, emission from the $\mathrm{Mg}$ II feature at $2800 \AA$ remains prominent, but the Fe II features become less defined. Also, for a few objects, only the emission peak near $2600 \AA$ is seen, without significant emission at $2800 \AA$ or $2400 \AA$ (see for example Gl 273, Gl 876 and Gl 682 in Figure 2b).

Although the prism data have very low resolution, we were able to extratct an estimate of the fluxes in the regions near the strong lines. The spectra were fit by four gaussians (centers held fixed at $\lambda=2385 \AA, 2560 \AA$, $2640 \AA$ and $2800 \AA$ ) and a second order polynomial. The area under the gaussians can then be used to characterize the flux due to emission features near those wavelengths. The result of one such fit is shown in Figure 3, and the fluxes measured for all spectra are listed in Table 2. If no flux is listed for a particular feature, that feature did not contribute significantly to the spectrum or the fit was unreliable.

\subsection{Comparison to Existing Data}

Approximately half of our observed sample have existing IUE low resolution spectra. Linsky et al. (1982) carried out an early pioneering study of ultraviolet emission in active cool stars with IUE, while a later comparative study of $\mathrm{Mg}$ II $\mathrm{h}$ and $\mathrm{k}$ emission with X-ray emission was undertaken by Mathioudakis \& Doyle (1989). However, the majority of stars observed with IUE are active, and the few lower activity objects with IUE spectra have relatively low $\mathrm{S} / \mathrm{N}$.

In order to better compare the IUE and ACS observations, we used the existing IUE spectra to simulate ACS spectra with SLIM. As previously discussed, the results for $\mathrm{AD}$ Leo are shown in Figure 1. The three peaks in the AD Leo ACS spectrum at $2400 \AA, 2600 \AA$ and $2800 \AA$ are recreated in the SLIM simulated spec-

\footnotetext{
${ }^{4}$ http://www.stecf.org/instruments/ACSgrism/slim/index.php
}

trum, and the fluxes in the data and simulated spectrum are a good match within the uncertainties. Not all of the SLIM simulated spectra gave such good results- many of the noisier IUE spectra produce accordingly noisier simulated spectra, so comparison between the simulated and observed fluxes is unreliable. As the best-exposed IUE spectra all had prominent $\mathrm{Mg}$ II emission, we chose to compare the Mg II flux between our ACS data and the IUE spectra as a test of our ability to measure accurate line fluxes. The flux in the ACS spectra due to the gaussian measured at $\lambda_{c}=2800 \AA$ was taken to be representative of the $\mathrm{Mg}$ II flux. The IUE $\mathrm{Mg}$ II lines were fit with a single gaussian as the $\mathrm{h} \& \mathrm{k}$ lines were blended in the low resolution data. Figure 4 shows IUE versus ACS Mg II surface fluxes, calculated using radii given in Reid \& Hawley (2005) Table 4.1, and distances from trigonometric parallax measurements. The brightest and most active stars, for which Mg II emission was most prominent, show a good match between the ACS and IUE measurements. Fainter objects were probably more affected by scattered light in the ACS spectra, and therefore are measured as having more flux than the IUE spectra.

Many of our stars have X-ray measurements from ROSAT. Figure 5 shows the surface ACS Mg II flux versus the ROSAT surface flux. Our results are very similar to Figure 1 from Mathioudakis \& Doyle (1989). Their flux-flux relation for dMe stars (stars with $\mathrm{H} \alpha$ in emission, represented as filled black circles) is overplotted as a dotted line. A slight positive offset exists between the Xray fluxes and the overplotted fit, as the Mathioudakis \& Doyle (1989) correlation used measurements from Einstein and EXOSAT rather than ROSAT [Linsky (1993)], but otherwise our results are in excellent agreement.

Most of the stars in our sample also have measured $\mathrm{H} \alpha$ equivalent widths, originally reported in Gizis, Reid, \& Hawley (2002) and included in Table 1 of this paper. Using the $\chi$ factor of Walkowicz et al. (2004), we calculated the flux in $\mathrm{H} \alpha$ for each star. Figure 6 shows the computed $\mathrm{H} \alpha$ flux versus the log of the ACS Mg II flux, where negative flux values imply that the $\mathrm{H} \alpha$ line is in absorption.

Taken together, Figures 5 and 6 present a complex picture of chromospheric and coronal emission. While the most "active" stars, such as AD Leo, have strong emission in $\mathrm{Mg}$ II, X-rays and $\mathrm{H} \alpha$, many of the stars possessing the highest amounts of $\mathrm{Mg}$ II emission (e.g. Gl 205 and Gl 299) have relatively weak X-ray emission and $\mathrm{H} \alpha$ in absorption. These results indicate that there is no simple relationship between the activity level (as indicated by $\mathrm{H} \alpha$ ) and where emission preferentially occurs, and the use of simple scaling relations between these line fluxes may be misleading.

\subsection{Comparison to Models}

We compare the near-UV observational data with predictions from the radiative hydrodynamic models of Allred et al. (2005, 2006). The models use solutions to the $1-\mathrm{D}$ equations of radiative hydrodynamics, including non-LTE radiative transfer in $\mathrm{H}, \mathrm{He}$ and $\mathrm{Ca}$ II, with flare heating provided by an electron beam. The results include predictions of atmospheric structure (temperature, density profiles), velocity and line profiles at many time steps during an episode of flare heating. We used the 
results of the Allred preflare and F10 models to investigate the Mg II and Fe II emission in and out of flaring states (the F10 model used here represents an average mid-flare atmosphere near a time step of $\sim 85$ sec; see Allred et al. (2006)). In addition we used two other preflare models, scaled to be either cooler or hotter at a given column mass than the Allred preflare atmosphere. The four atmospheric models are designated here as PF, Flare, LPLT (low pressure low temperature), and HPHT (high pressure high temperature), for the preflare, flare, cooler and hotter atmospheres, respectively. The model atmospheres are shown in Figure 7.

The Allred et al. models only include $\mathrm{H}$, $\mathrm{He}$ and $\mathrm{Ca}$ II in the detailed non-LTE treatment. However, thousands of other transitions (including those of $\mathrm{Mg}$ and $\mathrm{Fe}$ ) are included as background opacity in LTE. The solar models of Abbett \& Hawley (1999; see section 2.4) compare the effects of including detailed non-LTE calculations for $\mathrm{Mg}$ II or treating it as background opacity, and they find that there is little change in the total cooling rate for these two cases. Thus, including $\mathrm{Mg}$ and Fe in non-LTE is likely to make little difference in the overall energy balance of the atmosphere. However, since Allred et al. (2006) did not predict the Mg II or Fe II line profiles which comprise the majority of emission lines in our observed wavelength range, we analyzed our atmosphere models with the 'RH' non-LTE radiative transfer code described in Uitenbroek (2001). The RH code is based on the Multi-level Accelerated Lambda (MALI) formalism of Rybicki \& Hummer (1991, 1992), which allows both bound-bound and bound-free radiative transitions to overlap in wavelength. It also includes the effects of partial redistribution for strong bound-bound transitions such as Mg II h and $\mathrm{k}$. The Mg II calculations for the $\mathrm{PF}$ and Flare atmospheres were originally reported in Hawley et al. (2007). The non-LTE atomic level populations were calculated for a 9 level hydrogen atom with continua, an 11 level Mg II ion with continua and a 143 level Fe II ion with continuum. Other atomic transitions (of $\mathrm{He}, \mathrm{Ca}, \mathrm{C}, \mathrm{O}, \mathrm{Si}, \mathrm{Al}, \mathrm{NaI}$ and $\mathrm{N}$ ) and molecular transitions (of $\mathrm{H}_{2}+, \mathrm{C}_{2}, \mathrm{~N}_{2}, \mathrm{O}_{2}, \mathrm{CH}, \mathrm{CO}, \mathrm{CN}, \mathrm{NH}, \mathrm{NO} \mathrm{OH}$ and $\left.\mathrm{H}_{2} \mathrm{O}\right)$ are included as background opacity in LTE.

While the PR200L resolution is clearly insufficient for comparison with the detailed model line profiles, we can use these model spectra as inputs to SLIM, giving us PR200L simulated low resolution spectra for $\mathrm{Mg}$ II and Fe II. In Figures 8-11, we show the model spectra for $\mathrm{Mg}$ II and Fe II for each atmosphere, the SLIM simulated ACS spectra for Mg II and Fe II separately, and the combined result. All fluxes have been scaled to a star with the radius and distance of $\mathrm{AD}$ Leo $\left(\mathrm{R}=0.41 \mathrm{R}_{\text {Sun }}\right.$, $\mathrm{d}=4.9 \mathrm{pc}$ ) [Sciortino et al. (1999), Reid \& Hawley (2005)] to enable direct comparison to the data. Overall, the shape of the model spectra are qualitatively similar to the observed spectra. One notable feature is that the cooler atmospheres (LPLT and PF) do not predict a strong emission peak at $2800 \AA$, and instead predict a trough centered there. The resulting spectra are then reminiscent of the ACS data for less active stars, such as Gl 889.1 and Gl 825 (shown in Figure 2b). In every case, our models overpredict the Fe II emission. The result for our "Flare" atmosphere shows the strong Mg II emission at $2800 \AA$ that we see in many spectra (especially of the active stars), but the Fe II emission predicted by this same atmosphere vastly overwhelms the Mg II peak, which is not observed. We reproduce the observed peaks in the ACS data due to Fe II, though emission at the shorter wavelengths is stronger than predicted. We note, however, that in the IUE simulated spectrum of AD Leo (Figure 1), the Fe II emission at $2400 \AA$ was stronger than observed in the ACS spectrum- it therefore may be that the shortest wavelengths in the ACS spectra are not accurately measured as the signal is extremely faint.

\subsection{Specific Cases}

We used a combination of the resulting model prism spectra to match the ACS observations of Gl 876, Gl 825, Gl 889.1, Gl 273, and AD Leo. These specific stars were selected on the basis of their relatively well exposed spectra and variety of activity levels (as inferred by their $\mathrm{H} \alpha$ equivalent widths).In order to gain insight into the structure of the active regions of the chromosphere, we determine a "fill factor", or effective surface area coverage of which model spectrum best fits these stars. In several cases, the observed data are best fit by a combination of spectra from different atmospheres, indicating the probable existence of multiple components in the chromospheres of these stars. The best fits were determined by eye in each case, with preference toward matching the flux between $2200 \AA$ and $2900 \AA$. Wavelengths longward of $2900 \AA$ lie nearer the red pile-up, and therefore are more likely to be unreliable (for example, SLIM simulations of both models and IUE data never recreate the deep dip at $2950 \AA$, which is seen in all the ACS spectra).

\subsection{1. $G l \quad 876$ (dM4)}

Figure 12 shows the fit to the spectrum of Gl 876 . We find that the PF atmosphere provides the best fit to the observations, with a fill factor of $\sim 1.3 \%$. Gl 876 is of particular interest for astrobiology, as it is host to 3 known extrasolar planets, including a $\sim 7.5 \mathrm{M}_{\oplus}$ "super Earth". Gl 876 has an $\mathrm{H} \alpha$ absorption line $\left(\mathrm{EW}_{H \alpha}=-0.2 \AA\right)$, indicating that it possesses a low-to-intermediate level of chromospheric activity (as compared with $\mathrm{M}$ dwarfs with $\mathrm{H} \alpha$ lines in emission, which have a high level of chromospheric heating and are thus deemed "active"). M dwarfs with very little activity and those with moderate activity cannot be distinguished on the basis of $\mathrm{H} \alpha$ alone, as both weak and intermediate chromospheres show $\mathrm{H} \alpha$ in absorption [Cram \& Mullan (1985)]. However, information from other chromospheric lines (such as Ca II, $\mathrm{Mg}$ II and Fe II) may serve to break this degeneracy.

With $\mathrm{H} \alpha$ in absorption and a spectral type of dM4, Gl 876 is roughly equivalent in temperature and activity to the $3100 \mathrm{~K}$ inactive star used in the Segura et al. (2005) biomarker study. Segura et al. (2005) used a model spectrum from Allard et al. (1997) with no chromospheric flux for their cool, inactive M dwarf- however, it is debatable whether such a star exists, as most M dwarfs show evidence of a chromosphere at some level [Cram \& Mullan (1985)]. Low-to-intermediate activity stars, like Gl 876, are the true unknowns in studies of the effect of stellar flux on attendant planets- while they may not have the strong chromspheric emission of very active stars like $\mathrm{AD}$ Leo, their near-UV emission may still be non-neglible.

Scaling our spectrum of Gl 876 to the top-ofatmosphere flux for a planet in the habitable zone (semi- 
major axes given in Segura et al. (2005) Table 1), we find that the so-called "inactive" Gl 876 actually has an appreciable near-UV flux. In Figure 13, we plot our results for Gl 876 side by side with a similarly scaled spectrum of AD Leo (again using parameters from Segura et al. (2005) Table 1) and show that the expected near-UV flux at a planet in the habitable zone is actually comparable for both stars. Results for ozone production and methane chemistry in the atmosphere of a planet around Gl 876 would therefore be more in keeping with those given for $\mathrm{AD}$ Leo, rather than the predicted "methane runaway" in planets around quiescent M dwarfs [Segura et al. (2005)].

\subsection{2. $G l 273$ (dM3.5)}

In Figure 14, we plot the resulting model fit to our ACS spectrum of Gl 273. Here, the data are best fit by a combination of our LPLT and PF atmospheres in roughly equal portion $(0.6 \%$ and $0.5 \%$, respectively. This fit gives an overall fill factor of $\sim 1 \%$ for the active regions that produce the near-UV flux.

\subsection{3. $G l$ 825 (dKr)}

Gl 825 is one of the hotter, more massive stars in our sample. We find that a combination of the LPLT and $\mathrm{PF}$ atmosphere model spectra, at $3 \%$ and $4.5 \%$ fill factor respectively, provides a good match to the majority of the spectrum (Figure 15). There is an excess of flux at $\sim 2750$ that our model spectra do not reproduce, however, and increasing the fill factor to match this part of the spectrum then overestimates the Fe II flux at shorter wavelengths.

\subsubsection{Gl 889.1 (dM0)}

Gl 889.1 is best matched by a combination of the LPLT and HPHT atmospheres, at fill factors of $1.6 \%$ and $0.05 \%$ respectively. While the spectrum has a similar shape to that of Gl 825, Gl 889.1 has more short wavelength flux (from 2000 to $2200 \AA$ ) that is not well fit without the hotter HPHT component. The resulting fit is shown in Figure 16.

\subsubsection{AD Leo (dM3e)}

Fitting our results for AD Leo is challenging, as the models which match the Mg II emission clearly overpredict emission from Fe II. As some Fe II also contributes flux to the region around $2800 \AA$, a much smaller fill factor is necessary to match the combined Fe II $+\mathrm{Mg}$ II model spectra than what one would interpret from $\mathrm{Mg}$ II results alone. As AD Leo is a very active star, we first attempt to fit the data with our hottest "Flare" model (Figure 17, left panel). While a fill factor of $0.25 \%$ reproduces roughly the correct amount of flux around $2800 \AA$ and at wavelengths shorter than $2200 \AA$, the Fe II emission at $\sim 2400 \AA$ and $\sim 2600 \AA$ is much too large. The HPHT atmosphere (also at a fill factor of $0.25 \%$; Figure 17 , right panel) produces a much better match to these two Fe II features, but unfortunately does not account for any of the observed Mg II flux. Using the Mg II results for our Flare model alone, the emission near $2800 \AA$ is well matched with a fill factor of $1.6 \%$. While it is not physical to ignore the Fe II emission, the $1.6 \%$ coverage required to reproduce the $\mathrm{Mg}$ II flux provides an upper limit estimate on the area filled by this hot "Flare" component.

\section{CONCLUSIONS AND FUTURE WORK}

We obtained 31 ACS PR200L prism spectra of stars, covering spectral types K7 to M5, and including a variety of activity levels. The near-UV data presented here provide valuable constraints for both model stellar chromospheres and photochemical models of planetary atmospheres around M dwarfs.

We measured Mg II fluxes for our spectra and compared our results with complementary optical, near-UV and X-ray data. For those stars in our sample with IUE data, we used the IUE Mg II flux as a check of our ACS prism spectra measurements, and found them roughly consistent. We also examined the relationship between $\mathrm{Mg}$ II emission and ROSAT measurements of the X-ray flux. Our results agree with those of Mathioudakis \& Doyle (1989), showing a well-defined correlation for the most active dMe stars. Using existing $\mathrm{H} \alpha$ measurements for our sample in conjunction with the $\chi$ factor of Walkowicz et al. (2004), we computed $\mathrm{H} \alpha$ fluxes and compared these to our measured Mg II fluxes, finding a complex relationship between the optical and nearUV emission. Together, the $\mathrm{Mg}$ II, X-ray and $\mathrm{H} \alpha$ data discussed here present a complex picture of the chromospheric and coronal emission in these stars.

Using the 'RH' NLTE radiative transfer code, we calculated Mg II and Fe II spectra for a suite of model atmospheres. The resulting model spectra were then degraded to the resolution of the ACS prism data using SLIM, the ACS prism spectra simulator. We compared our results with several of the observed spectra to infer potential chromospheric structure and active region coverage, finding that most stars could be matched by an active region area coverage of a few percent of the stellar surface. This result is consistent with our findings in Hawley et al. (2007), where we determined that $\sim 0.5 \%$ of the visible stellar surface was involved in the observed flares. While in many cases the qualitative shape of the model spectra matched the observations, Fe II was overpredicted for the most active objects. The empirical results we find for $\mathrm{Mg}$ II, X-ray and $\mathrm{H} \alpha$ pose an interesting challenge for the models. The model atmospheres described in this paper will be used in future calculations of $\mathrm{H} \alpha$ and $\mathrm{Ca}$ II K, which will then be compared with our results for the near-UV emission.

In the case of Gl 876, a dM4 star that hosts three extrasolar planets, we scaled our observed spectra to match the expected flux at the distance of a planet in the habitable zone. We compared our results to a similar scaling of our AD Leo observation, and then discussed these results in the light of previous studies of M dwarf planet habitability. We find that our observation of what might be called an "inactive" star (on the basis of $\mathrm{H} \alpha$, the usual proxy for activity in late type stars) actually shows a substantial amount of near-UV flux. Although Gl 876 is a lower luminosity and lower activity star than AD Leo, the non-negligible near-UV flux and proximity of the habitable zone conspire to make it a similar host for planets. The spectra described in this paper will be further used as input stellar flux for the Virtual Planet Laboratory's photochemical planetary atmosphere models. 
The authors thank Pat Hartigan for his guidance with the reduction process. LMW thanks Han Uitenbroek for the use of his RH code and Mats Carlsson for his valuable input. LMW and SLH thank the Helen Riaboff Whiteley Center at the University of Washington Friday Harbor Laboratories, in whose phronistery this paper was completed. Support for program 10525 was provided by
NASA through a grant from the Space Telescope Science Institute, which is operated by the Association of Universities for Research in Astronomy, Inc., under NASA contract NAS 5-26555. This research has made use of the aXe extraction software, produced by ST-ECF, Garching, Germany.

\section{REFERENCES}

Abbett, W. P., \& Hawley, S. L. 1999, ApJ, 521, 906

Allard, F., Hauschildt, P. H., Alexander, D. R., \& Starrfield, S. 1997, ARA\&A, 35, 137

Allred, J.C. et al. 2002, BAAS, 34, 1113 (abstract 12.05)

Allred, J. C., Hawley, S. L., Abbett, W. P., \& Carlsson, M. 2005, ApJ, 630, 573

Allred, J. C., Hawley, S. L., Abbett, W. P., \& Carlsson, M. 2006 , ApJ, 644, 484

Andreeshchev, A., Scalo, J., \& Smith, D. (2002) Very low mass stars as optimum sites of habitable planets and their detection. 2002, International Journal of Astrobiology, 1, 87

Burgasser, A.J., Liebert, J., Kirkpatrick, J.D., \& Gizis, J.E. 2002, AJ, 123, 2744

Carlsson, M. 1986, Uppsala Astron. Obs. Report, No. 33

Carlsson, M. \& Stein, R.F. 1997, ApJ, 481, 500

Cram, L. E., \& Mullan, D. J. 1985, ApJ, 294, 626

Fleming, T.A., Giampapa, M.S., \& Schmitt, J.H.M.M. 2000, ApJ 533,372

Gizis, J.E. et al. 2000, AJ, 120, 1085

Gizis, J.E., Reid, I.N., \& Hawley, S.L. 2002, AJ, 123, 3356

Hauschildt et al. 1999, ApJ, 525, 871

Hawley, S.L., Gizis, J.E., \& Reid, I.N. 1996, AJ, 112, 2799

Hawley, S.L., Reid, I.N., \& Tourtellot, J.G. 2000, in Very Low-mass Stars and Brown Dwarfs, ed. R. Rebolo and M.R. ZapateroOsorio, Cambridge University Press, UK, pg. 109

Hawley, S.L. et al. 2002, BAAS, 34, 1125 (abstract 16.04)

Hawley, S. L., Walkowicz, L. M., Allred, J. C., \& Valenti, J. A. 2007, PASP, 119, 67

Hawley, S.L. \& Johns-Krull, C.M. 2003, ApJ, 588, L109- L112

Hawley, S. L. \& Pettersen, B. R. 1991, ApJ, 378, 725

Hawley, S. L., et al. 2003, ApJ, 597, 535

Johns-Krull, C. M., Hawley, S. L., Basri, G., \& Valenti, J. A. 1997, ApJS, 112, 22

Joshi, M. M., Haberle, R. M., \& Reynolds, R. T. 1997, Icarus, 129, 450

Joshi, M. 2003, Astrobiology, 3, 415

Joy, A.H. \& Abt, H.A. 1974, ApJS, 28, 1
Liebert, J., Kirkpatrick, J.D., Reid, I.N., Fisher, M.D. 1999, ApJ, 519,345

Linsky, J. L. 1993, Astrophysics and Space Science Library, Proceedings of a conference, held in Palermo, Italy, 22-26 June 1992, Dordrecht: Kluwer, —c1993, edited by Linsky, Jeffrey L.,

Linsky, J.L., Wood, B.E., Brown, A., Giampapa, M.S., \& Ambruster, C. 1995, ApJ, 455, 670

Mathioudakis, M. \& Doyle, J. G. 1989, A\&A, 224, 179

Mathioudakis, M. \& Doyle, J.G. 1992, A\&A, 262, 523

Mohanty, S. \& Basri, G. 2002, ApJ, 571, 469

Osten, R. A., Hawley, S. L., Allred, J. C., Johns-Krull, C. M., \& Roark, C. 2005, ApJ, 621, 398

Reid, I.N., Hawley, S.L., \& Gizis, J.E. 1995, AJ, 110, 1838

Reid, I. N., \& Hawley, S. L. 2005, New Light on Dark Stars Red Dwarfs, Low-Mass Stars, Brown Stars, by I.N. Reid and S.L. Hawley. Springer-Praxis books in astrophysics and astronomy. Praxis Publishing Ltd, 2005. ISBN 3-540-25124-3,

Rutledge, R.E., Basri, G., Martin, E.L., Bildsten, L. 2000, ApJ, 538, L141

Rybicki, G. B., \& Hummer, D. G. 1991, A\&A, 245, 171

Rybicki, G. B., \& Hummer, D. G. 1992, A\&A, 262, 209

Sciortino, S., Maggio, A., Favata, F., \& Orlando, S. 1999, A\&A, 342,502

Segura, A. et al. 2003, Astrobiology, 3, 689

Segura, A., Kasting, J. F., Meadows, V., Cohen, M., Scalo, J., Crisp, D., Butler, R. A. H., \& Tinetti, G. 2005, Astrobiology, 5, 706

Tarter et al. 2007, Astrobiology, 7, 1

Udry, S., et al. 2007, A\&A, 469, L43

Uitenbroek, H. 2001, ApJ, 557, 389

van den Oord, G. H. J., et al. 1996, A\&A, 310, 908

Walkowicz, L. M., Hawley, S. L., \& West, A. A. 2004, PASP, 116 , 1105

West, A. A., et al. 2004, AJ, 128, 426 
TABLE 1

Observed Sample

\begin{tabular}{|c|c|c|c|c|c|c|c|}
\hline HST Name & Name & $\mathrm{NN}$ & $\mathrm{M}_{v}^{\mathrm{a}}$ & $\mathrm{H} \alpha^{\mathrm{a}}$ & $\mathrm{d}(\mathrm{pc})^{\mathrm{a}}$ & Sp. Type ${ }^{\mathrm{a}}$ & Other NUV Obs. \\
\hline LP $476-207$ & GJ 3322 & 853 & 12.41 & 6.03 & 6.5 & $\mathrm{M} 4.0 \mathrm{Ve}$ & \\
\hline V AD LEO & Gl 388 & 1616 & 10.96 & 3.40 & 4.9 & $\mathrm{M} 3.0 \mathrm{Ve}$ & IUE \\
\hline LHS 191 & GJ 3289 & 773 & 17.16 & 2.60 & 17.0 & M6.5V & $\cdots$ \\
\hline LHS 3376 & GJ 4053 & 2897 & 14.09 & 2.14 & 7.5 & $\mathrm{M} 4.5 \mathrm{Ve}$ & $\cdots$ \\
\hline V 1285 AQL & Gl 735 & 2976 & 10.09 & 2.10 & 10.1 & $\mathrm{M} 3.0 \mathrm{Ve}$ & $\cdots$ \\
\hline LP $771-95$ & GJ 3192 & 538 & 11.93 & 0.93 & 6.4 & $\mathrm{M} 3.5 \mathrm{Ve}$ & $\cdots$ \\
\hline LHS 252 & GJ 3512 & 1355 & 15.08 & 0.31 & 9.9 & $\mathrm{M} 5.5 \mathrm{Ve}$ & $\cdots$ \\
\hline V DP DRA & Gl 487 & 2017 & 11.28 & 0.20 & 8.4 & $\mathrm{M} 3.0 \mathrm{Ve}$ & $\cdots$ \\
\hline $\mathrm{BD}+012447$ & Gl 393 & 1642 & 10.32 & -0.53 & 7.3 & $\mathrm{M} 2.0 \mathrm{~V}$ & $\cdots$ \\
\hline HD 42581 & Gl 229 & 1021 & 9.38 & -0.51 & 5.7 & $\mathrm{M} 0.5 \mathrm{~V}$ & IUE \\
\hline HD 199305 & Gl 809 & 3263 & 9.20 & -0.46 & 7.4 & $\mathrm{M} 0.5 \mathrm{~V}$ & IUE \\
\hline HD 119850 & Gl 526 & 2176 & 9.79 & -0.45 & 5.4 & $\mathrm{M} 1.5 \mathrm{~V}$ & $\ldots$ \\
\hline HD 165222 & Gl 701 & 2859 & 9.85 & -0.45 & 8.1 & M1.0V & $\cdots$ \\
\hline $\mathrm{BD}+66717$ & Gl 424 & 1770 & 9.56 & -0.44 & 8.9 & M0.0V & IUE \\
\hline $\mathrm{BD}+112576$ & Gl 514 & 2123 & 9.74 & -0.43 & 7.3 & $\mathrm{M} 0.5 \mathrm{~V}$ & $\cdots$ \\
\hline HD 216899 & Gl 880 & 3616 & 9.55 & -0.42 & 6.7 & $\mathrm{M} 1.5 \mathrm{~V}$ & $\cdots$ \\
\hline $\mathrm{BD}+7068 \mathrm{~b}$ & Gl 48 & 195 & 10.52 & -0.40 & 8.0 & M3.0V & $\cdots$ \\
\hline HD 36395 & Gl 205 & 930 & 9.19 & -0.40 & 5.7 & M1.5 & IUE \\
\hline Gl 889.1 & . & 3637 & 9.76 & -0.39 & 17.0 & $\mathrm{M} 0.0 \mathrm{~V}$ & $\cdots$ \\
\hline $\mathrm{BD}+183421$ & Gl 686 & 2792 & 10.14 & -0.38 & 7.9 & M1.0V & $\cdots$ \\
\hline GJ 2066 & . & 1299 & 10.38 & -0.36 & 8.8 & $\mathrm{M} 2.0 \mathrm{~V}$ & $\cdots$ \\
\hline $\mathrm{BD}+68946$ & Gl 687 & 2797 & 10.85 & -0.34 & 4.6 & M3.0V & IUE \\
\hline $\mathrm{BD}+014774$ & Gl 908 & 3759 & 10.20 & -0.33 & 5.7 & M1.0V & IUE \\
\hline $\mathrm{BD}+051668$ & Gl 273 & 1168 & 11.97 & -0.30 & 3.8 & M3.5V & IUE \\
\hline $\mathrm{BD}+61195$ & Gl 49 & 199 & 9.84 & -0.28 & 8.8 & $\mathrm{M} 1.5 \mathrm{~V}$ & IUE \\
\hline $\mathrm{BD}+442051$ & Gl $412 \mathrm{~A}$ & 1723 & 10.06 & -0.26 & 5.4 & $\mathrm{M} 0.5 \mathrm{~V}$ & IUE \\
\hline V $2306 \mathrm{OPH}$ & Gl 628 & 2599 & 12.02 & -0.23 & 4.1 & $\mathrm{M} 3.5 \mathrm{~V}$ & $\ldots$ \\
\hline V IL AQR & Gl 876 & 3604 & 11.84 & -0.20 & 4.6 & $\mathrm{M} 4.0 \mathrm{~V}$ & IUE \\
\hline BD -40 9712 & Gl 588 & 2446 & 10.45 & $\cdots$ & 5.9 & $\mathrm{M} 2.5 \mathrm{~V}$ & $\ldots$ \\
\hline BD -44 11909 & Gl 682 & 2779 & 12.54 & $\cdots$ & 4.8 & M3.5V & $\cdots$ \\
\hline HD 33793 & Gl 191 & 887 & 10.56 & $\cdots$ & 4.6 & M1.0V & IUE \\
\hline HD 202560 & Gl 825 & 3326 & 8.74 & $\ldots$ & 3.9 & $\mathrm{~K} 7 \mathrm{~V}$ & IUE \\
\hline HD 191849 & Gl 784 & 3174 & 8.99 & $\cdots$ & 6.3 & $\mathrm{~K} 7 \mathrm{~V}$ & IUE \\
\hline $\mathrm{BD}-515974$ & Gl 438 & 1832 & 10.36 & $\cdots$ & 10.0 & $\mathrm{M} 0.0 \mathrm{~V}$ & $\cdots$ \\
\hline
\end{tabular}

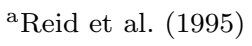


TABLE 2

Measured Fluxes

\begin{tabular}{|c|c|c|c|c|}
\hline Name & $\mathrm{F}_{\lambda 2385}$ & $\begin{array}{c}\mathrm{F}_{\lambda 2560} \\
10^{-14} \text { erg }\end{array}$ & $\begin{array}{l}\mathrm{F}_{\lambda 2640} \\
\mathrm{~s} \mathrm{~s}^{-1} \mathrm{~cm}^{-2}\end{array}$ & ${ }_{2} \mathrm{~F}_{\lambda 2800}$ \\
\hline GJ 3322 & 4.57 & 2.40 & 31.03 & 13.77 \\
\hline GJ 4053 & & 2.89 & 0.06 & 0.99 \\
\hline Gl 735 & 18.28 & 97.96 & 18.41 & 66.92 \\
\hline GJ 3192 & $\begin{array}{r}10.20 \\
3.83\end{array}$ & 56.72 & $\begin{array}{r}10.41 \\
0.00\end{array}$ & 3.93 \\
\hline GJ 3512 & 0.01 & 0.07 & 1.35 & $\ldots$ \\
\hline Gl 487 & 0.24 & 22.20 & 48.30 & 7.26 \\
\hline Gl 908 & & 37.70 & 82.20 & 18.66 \\
\hline Gl 229 & 228.77 & 97.82 & 540.20 & 186.81 \\
\hline Gl 809 & 135.85 & 1391.10 & 611.09 & 108.61 \\
\hline Gl 686 & 86.84 & & 213.42 & 39.94 \\
\hline Gl 526 & $\ldots$ & 302.39 & 202.02 & 76.30 \\
\hline Gl 701 & 252.53 & 101.87 & 145.01 & 13.44 \\
\hline Gl 424 & $\ldots$ & $\ldots$ & 102.39 & 24.87 \\
\hline Gl 514 & 14.21 & 0.004 & 288.69 & 74.54 \\
\hline Gl 880 & 5.02 & 81.50 & $\ldots$ & 86.72 \\
\hline Gl 48 & $\ldots$ & 132.56 & 556.20 & $\ldots$ \\
\hline Gl 205 & 181.91 & $\ldots$ & 803.12 & 222.18 \\
\hline GL 889.1 & 28.52 & 14.53 & 37.83 & 5.48 \\
\hline GJ 2066 & 4.23 & 31.99 & 59.10 & 14.70 \\
\hline Gl 687 & $\ldots$ & 184.98 & 24.50 & \\
\hline Gl 273 & $\ldots$ & 127.47 & 50.31 & 30.36 \\
\hline Gl 49 & 33.69 & & 141.92 & 32.84 \\
\hline Gl 412 & 118.46 & 113.57 & 215.92 & 27.42 \\
\hline Gl 876 & & 27.66 & 92.67 & 8.18 \\
\hline Gl 588 & 115.89 & $\ldots$ & 185.24 & $\ldots$ \\
\hline Gl 682 & 1.06 & & 55.76 & \\
\hline Gl 191 & $\ldots$ & 337.53 & 166.35 & 33.22 \\
\hline Gl 825 & 931.56 & $\ldots$ & 2616.64 & 201.13 \\
\hline Gl 784 & 1210.34 & 78.76 & 558.03 & 110.58 \\
\hline Gl 438 & 0.00 & & 59.72 & 21.06 \\
\hline Gl 388 & 29.05 & 175.22 & 55.45 & 126.99 \\
\hline
\end{tabular}



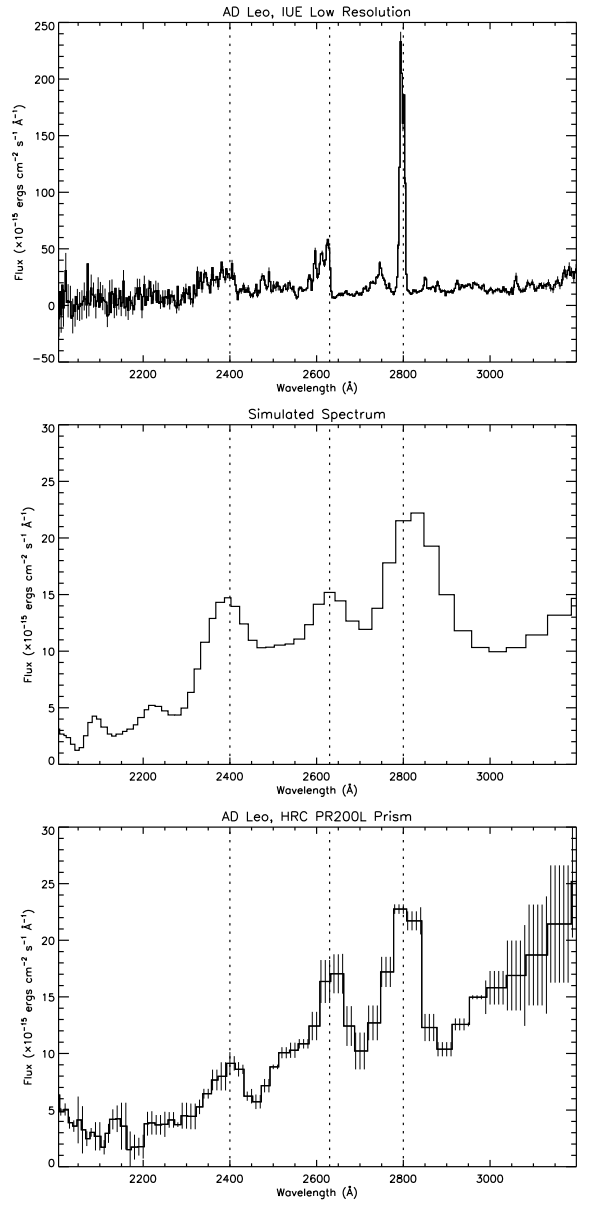

FIG. 1.- AD Leo as observed by IUE (top), prism spectrum simulated from IUE using SLIM (middle), and as observed with the HRC PR200L. 
Walkowicz et al.
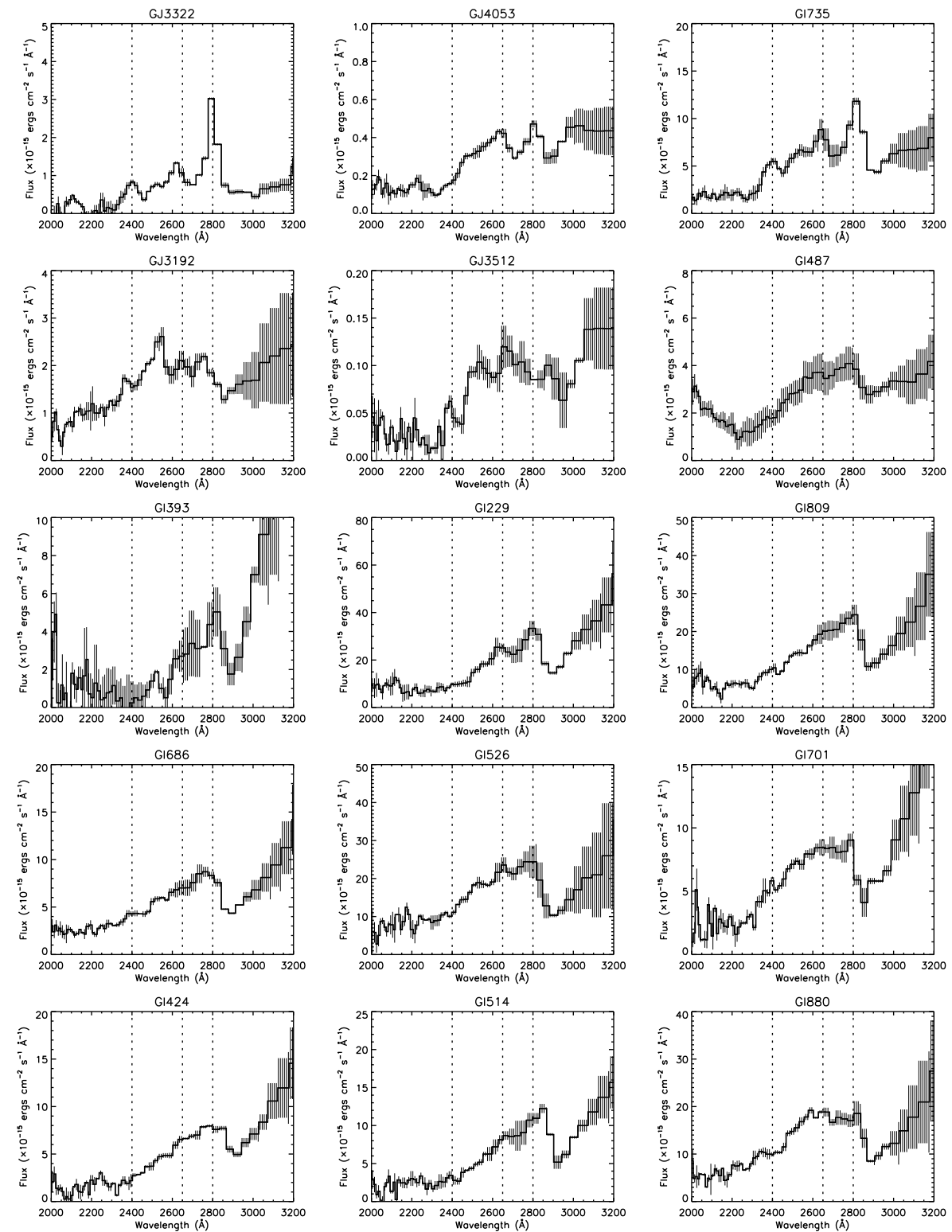

FIG. 2A.- ACS PR200L prism spectra. 

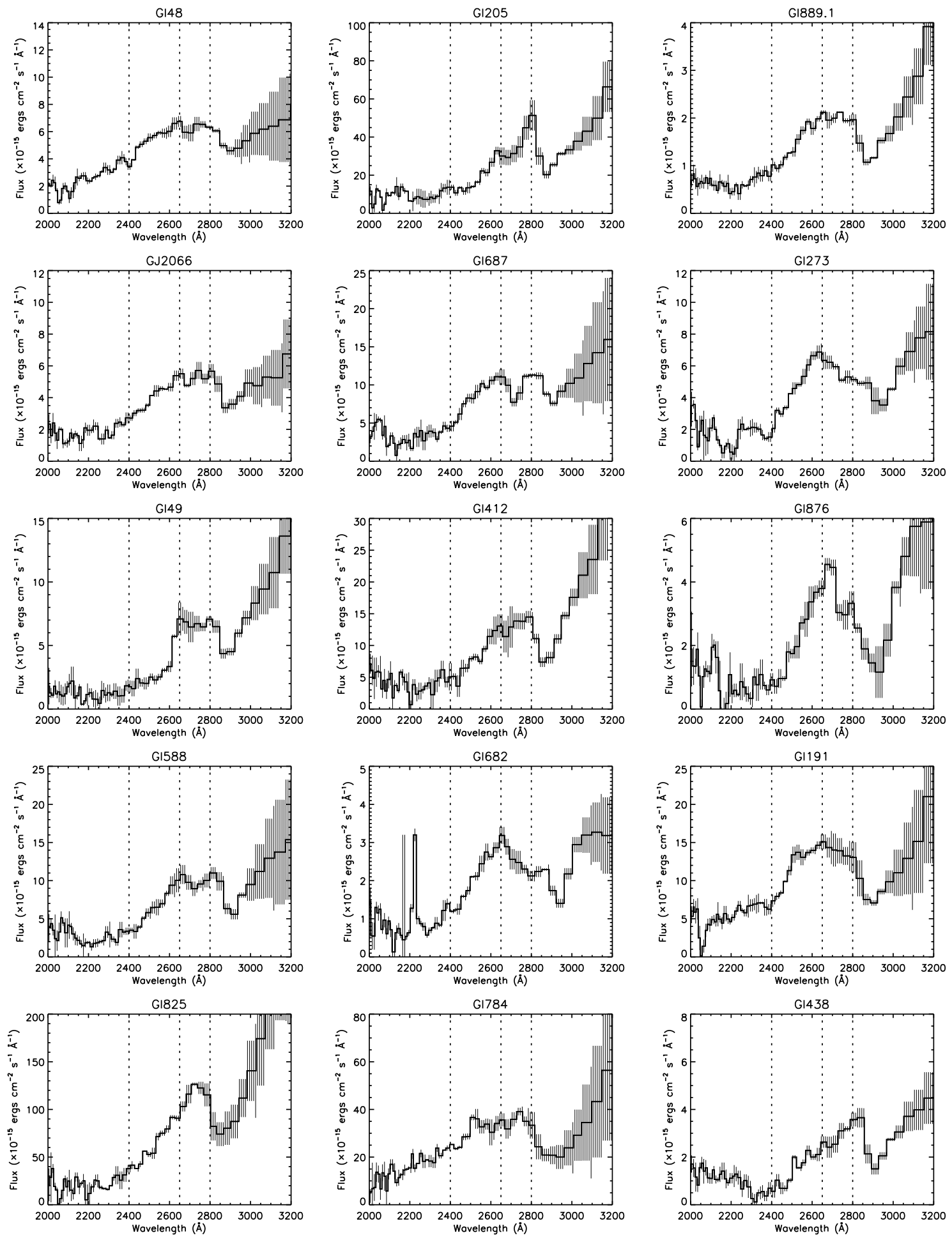

Fig. 2b. - Continued. 


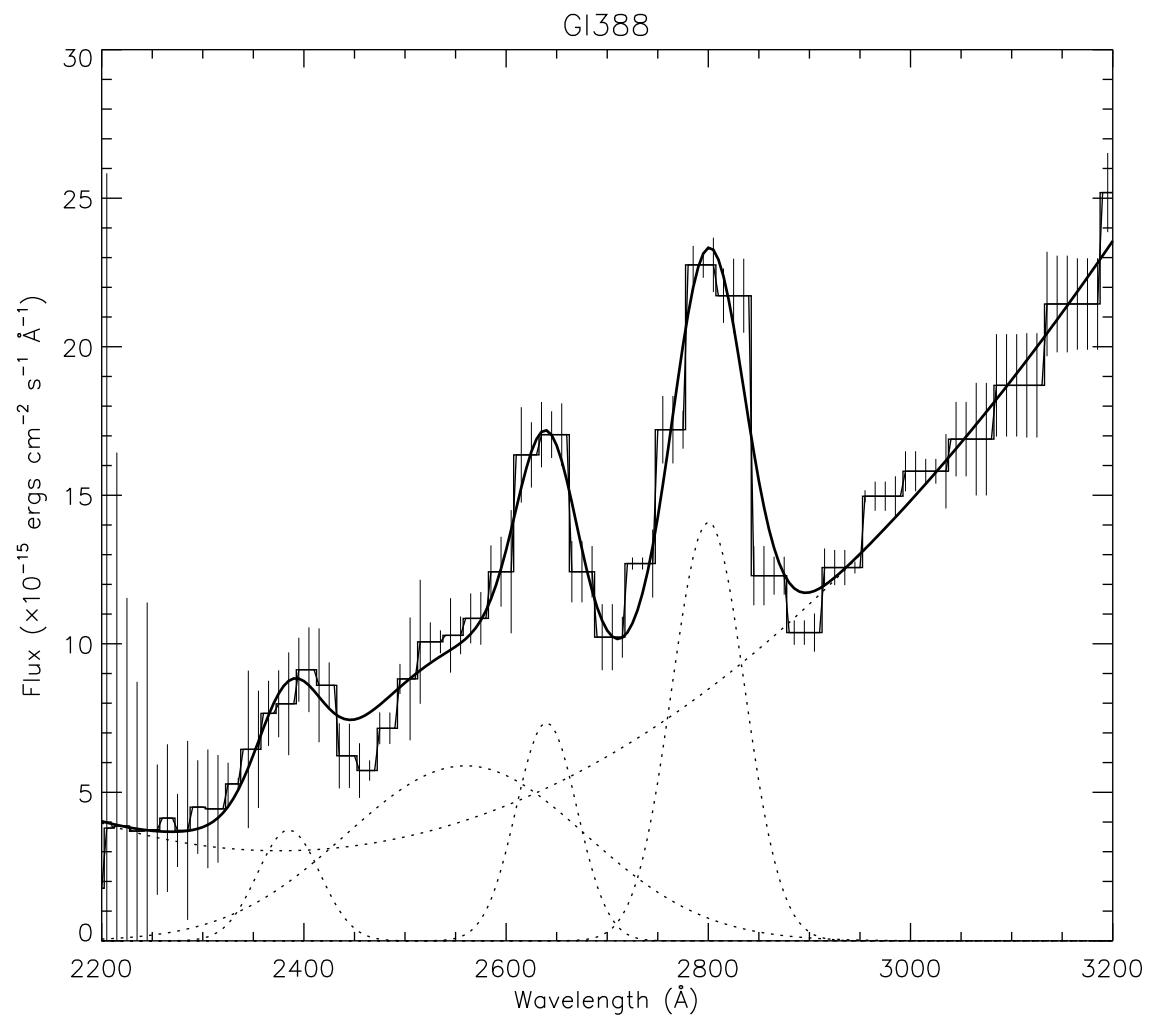

FIG. 3.- Example fit of ACS prism spectra, shown here for AD Leo. The three relatively narrow gaussians represent fits to the clusters of Fe II lines at 2400 and $2600 \AA$ and to the $\mathrm{Mg}$ II h and k lines at $2800 \AA$. The broad gaussian centered near $2500 \mathrm{~A}$ is used to fit a collection of unresolved lines between the two main features at 2400 and $2600 \AA$, together with the slowly varying second-order polynomial, which is used to fit the background component of unresolved lines and continuum edges throughout the spectrum.

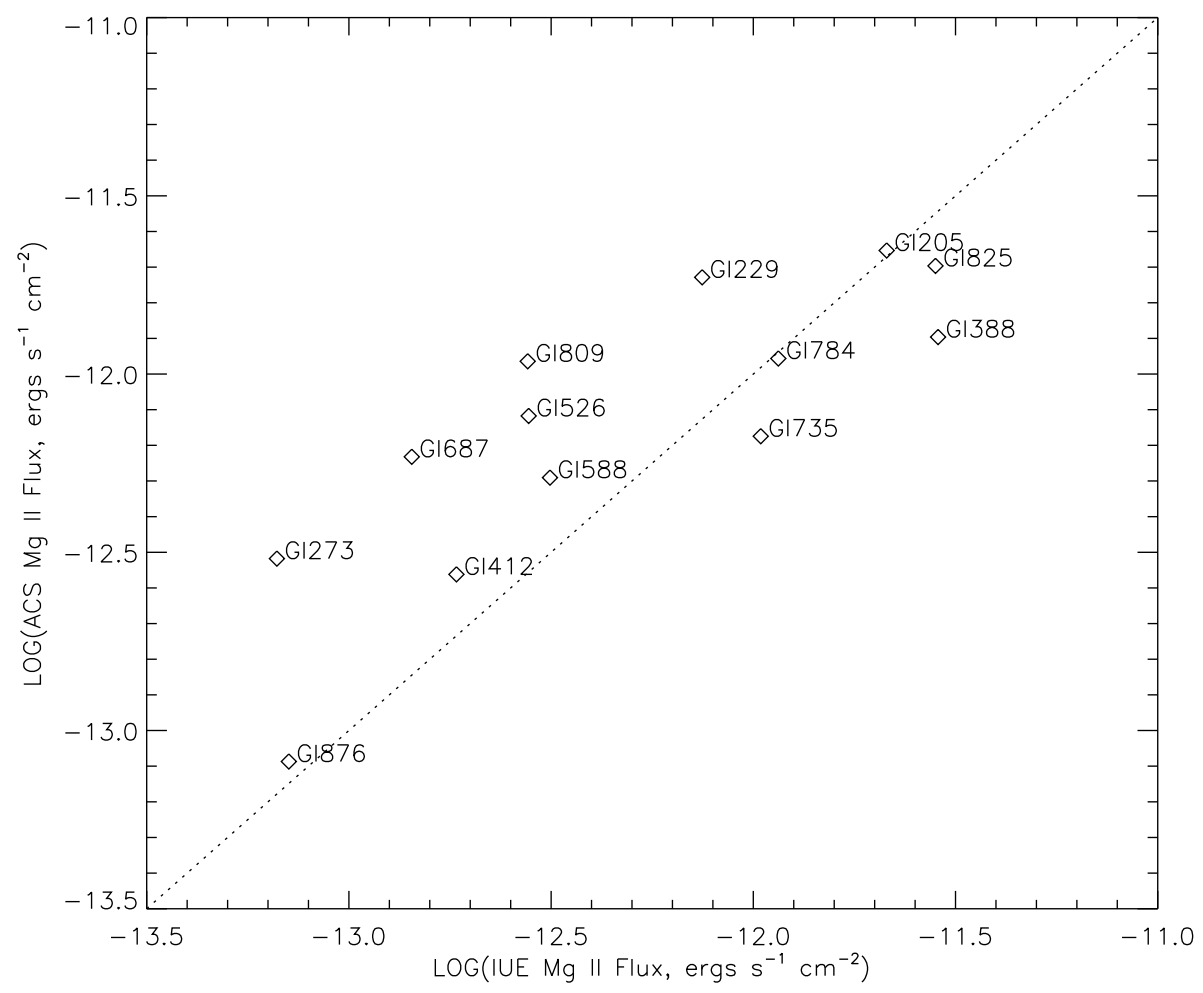

FIG. 4.- Comparison of Mg II surface fluxes measured from ACS and IUE spectra. The dotted line indicates equal fluxes from both instruments. The most active stars (e.g. Gl 388, Gl 735 and Gl 205) tend to live on or below the line, while less active stars show higher ACS fluxes, probably due to contamination from scattered light. 


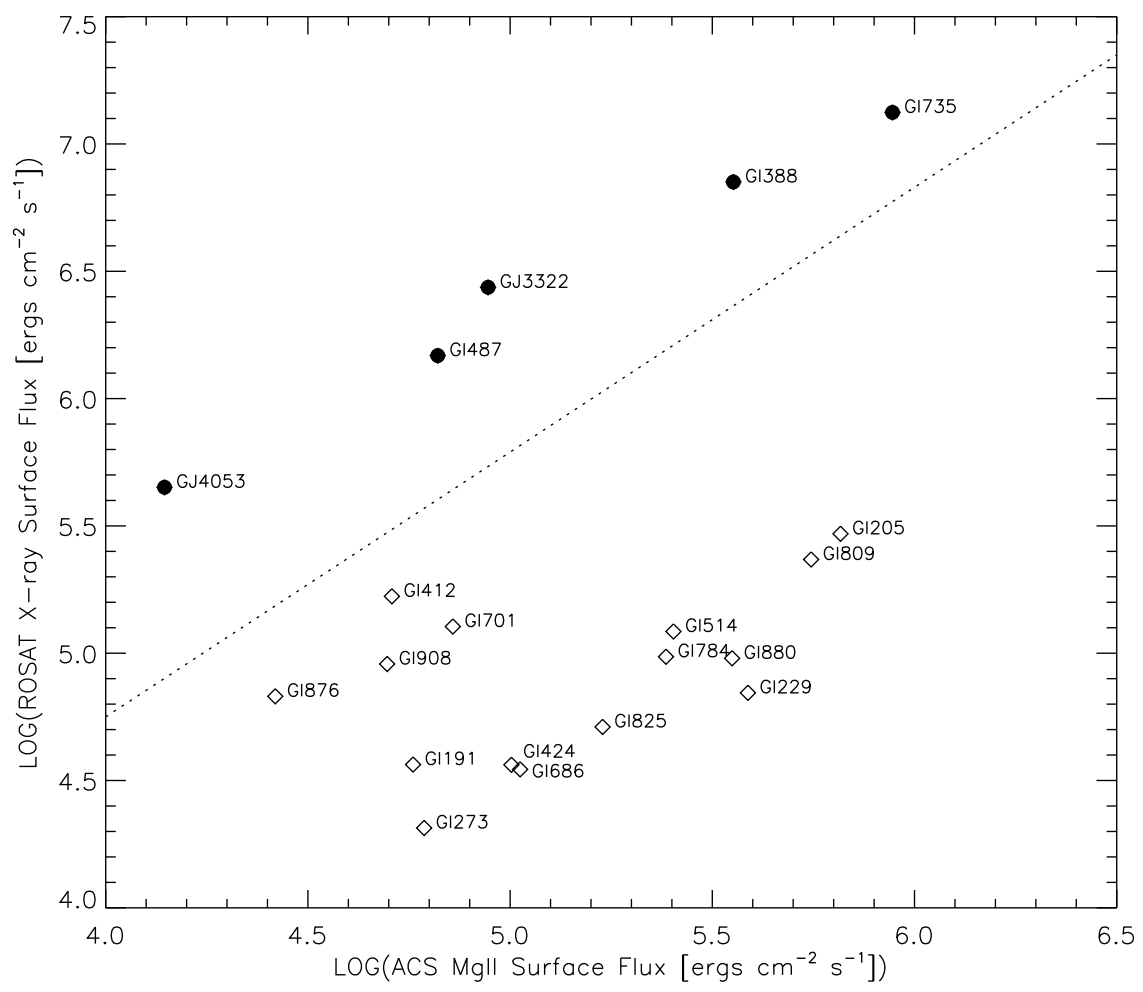

FIG. 5.- Comparison of X ray fluxes from ROSAT with Mg II measured from ACS spectra. The dotted line represents the fit to dMe stars from Mathioudakis \& Doyle (1989). A slight offset exists between their fit and the dMe stars in our sample (plotted as filled circles), as Mathioudakis \& Doyle (1989) used X-ray fluxes from Einstein and EXOSAT, while we compare with ROSAT, but otherwise our results are in excellent agreement with the fit.

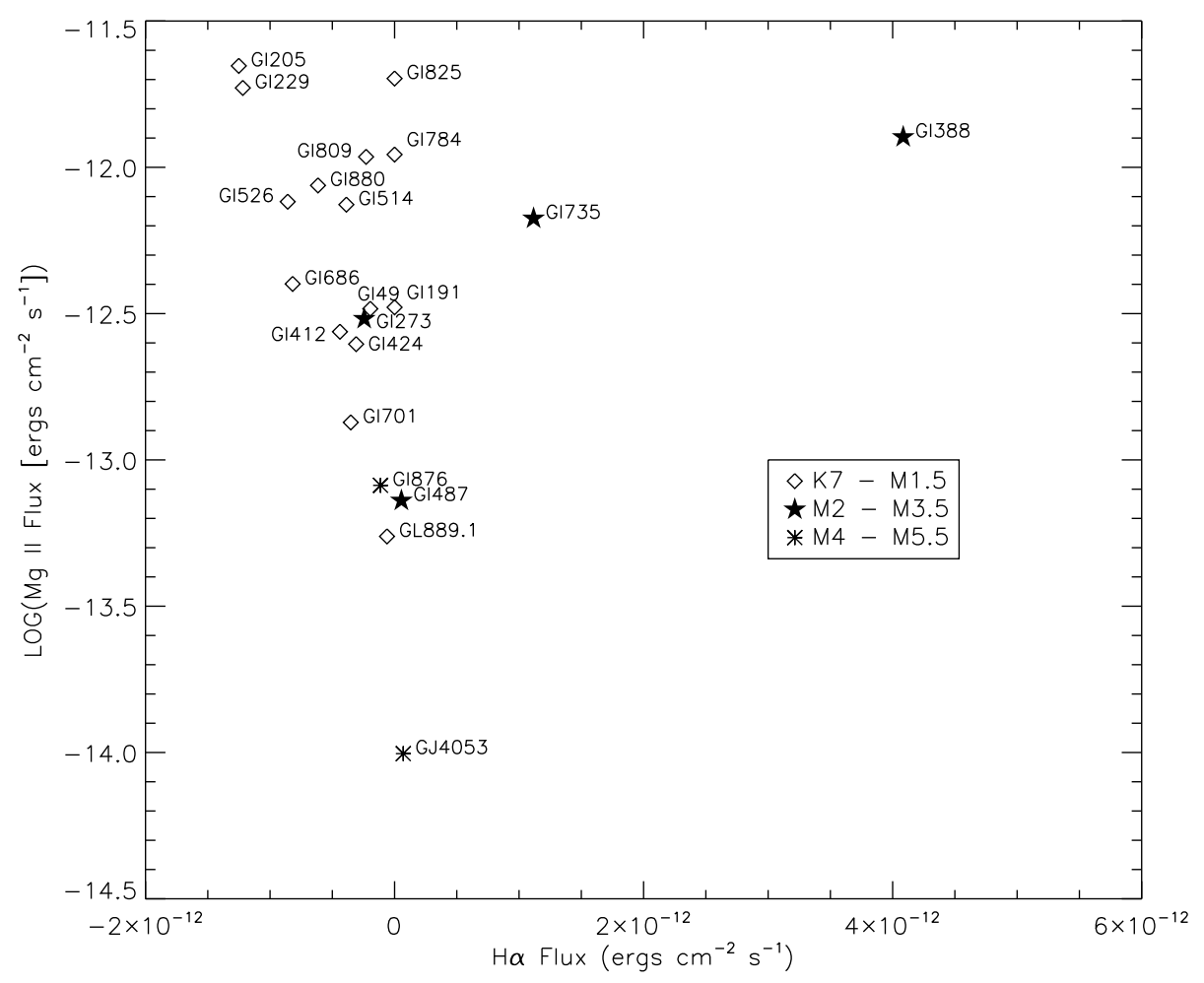

FIG. 6.- Comparison of $\mathrm{Mg}$ II measured from ACS spectra with $\mathrm{H} \alpha$ fluxes calculated using measured $\mathrm{H} \alpha$ equivalent widths and the $\chi$ factor relation of Walkowicz et al. (2004). H $\alpha$ fluxes are shown on a linear scale to indicate which are in absorption (negative fluxes) and which are in emission (positive flux). 


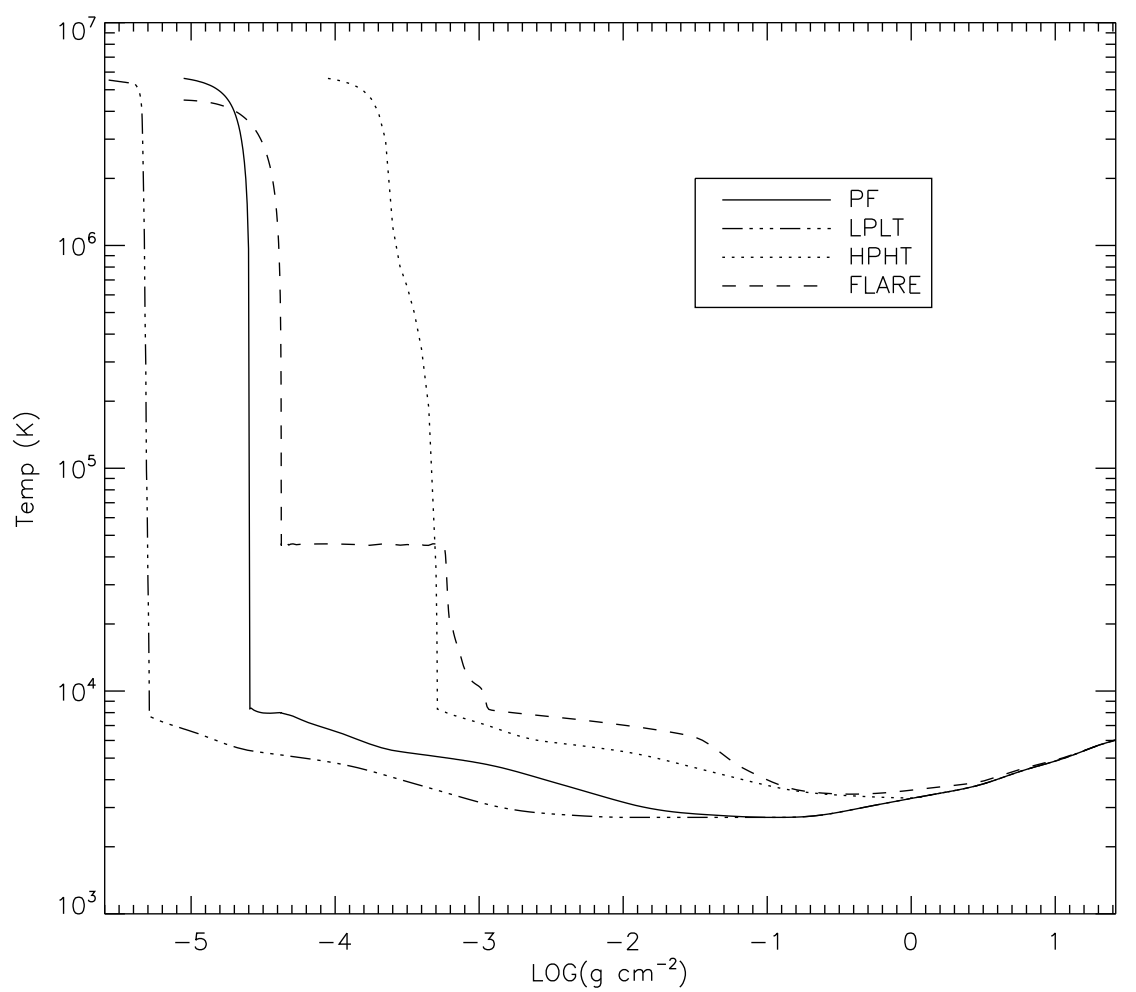

Fig. 7.- Model atmospheres used for Mg II and Fe II calculations. The PF (preflare), LPLT (low temperature low pressure) and HPHT (high pressure high temperature) models all represent quiescent atmospheres at different scalings, while the Flare atmosphere is a snapshot at $\sim 85 \mathrm{sec}$ from the hydrodynamic models of Allred et al. (2005). 

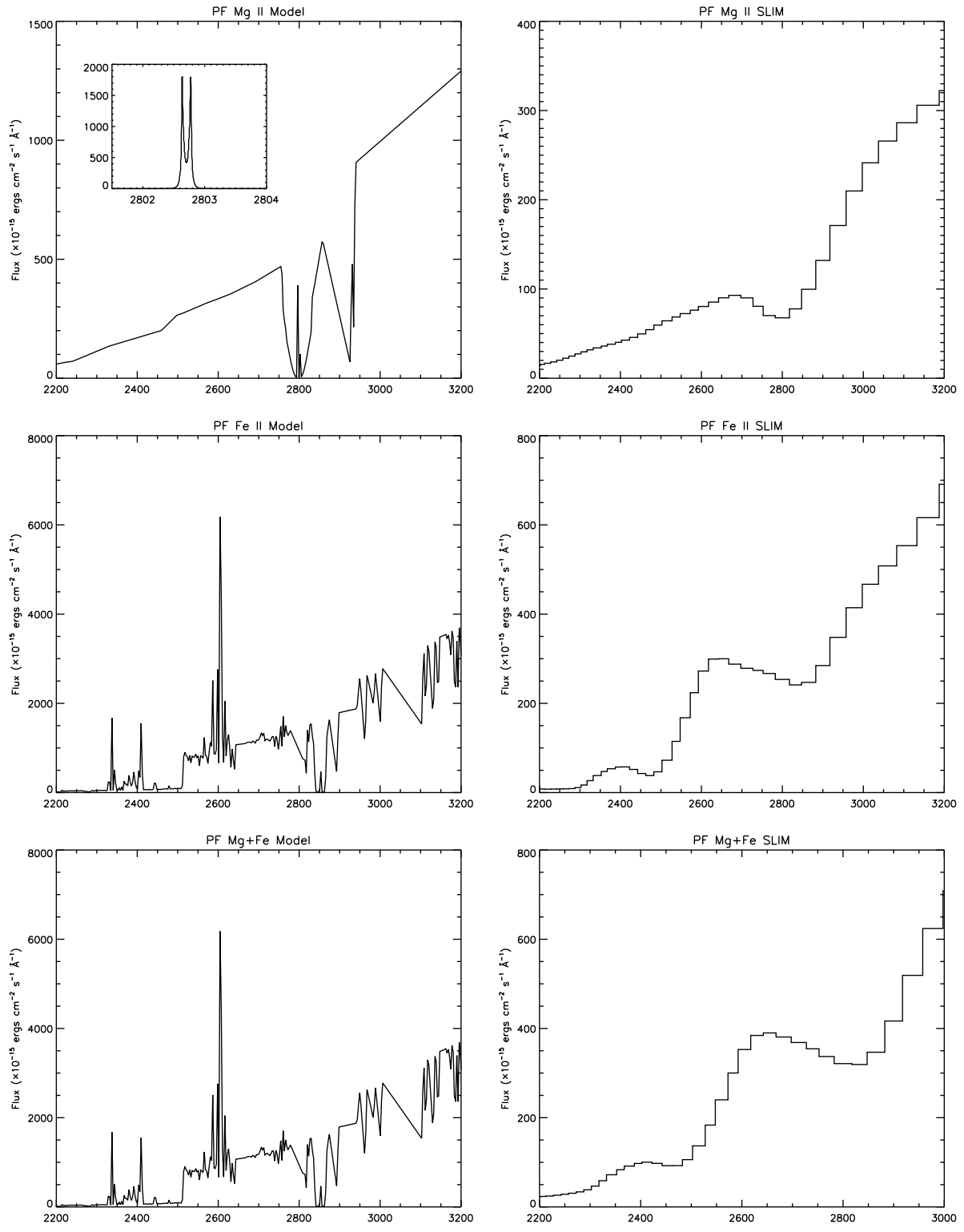

FIG. 8.- Mg II and Fe II Model results for PF atmosphere, degraded to ACS PR200L resolution using SLIM. 
Walkowicz et al.
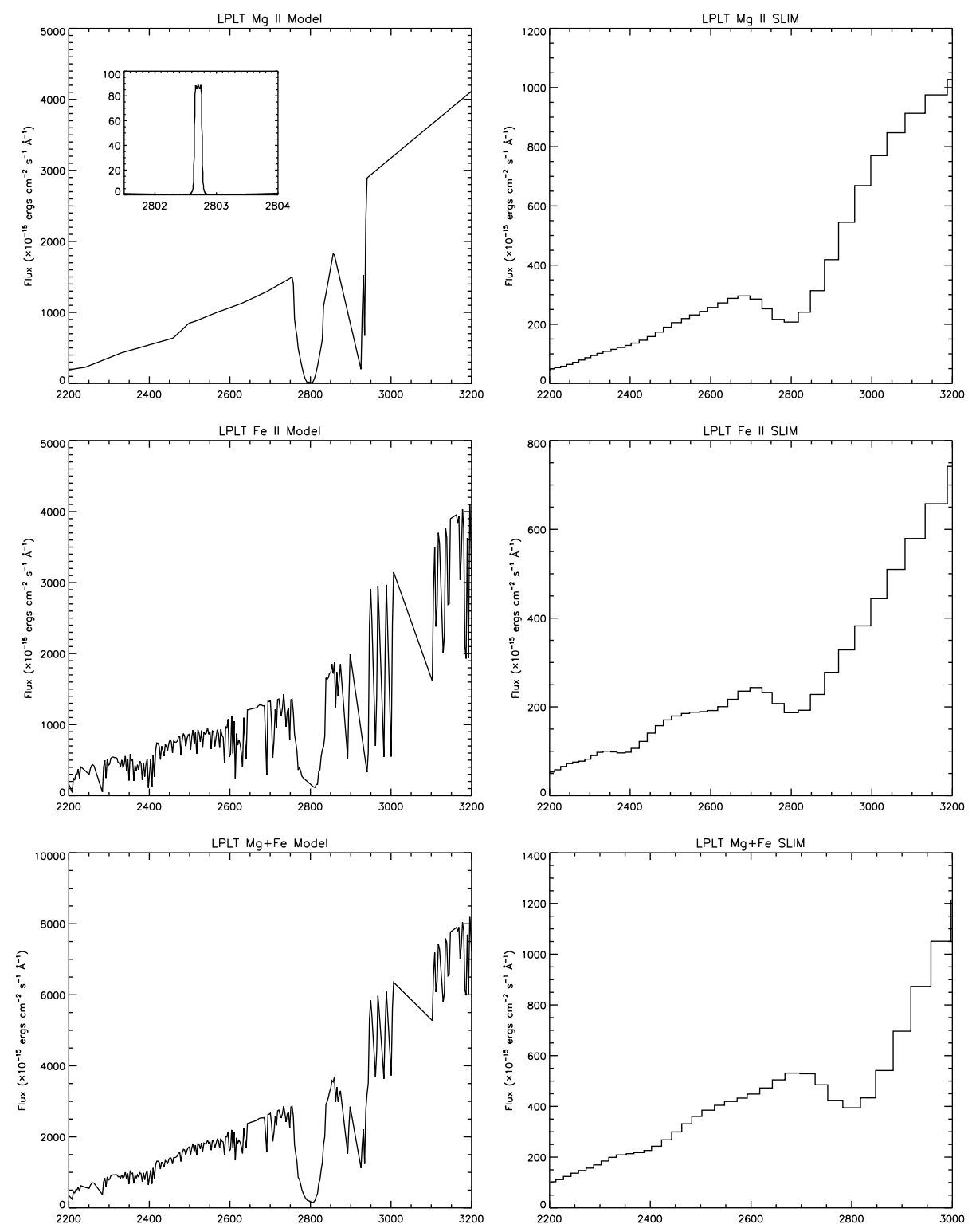

FIG. 9.- Mg II and Fe II Model results for LPLT atmosphere, degraded to ACS PR200L resolution using SLIM. 

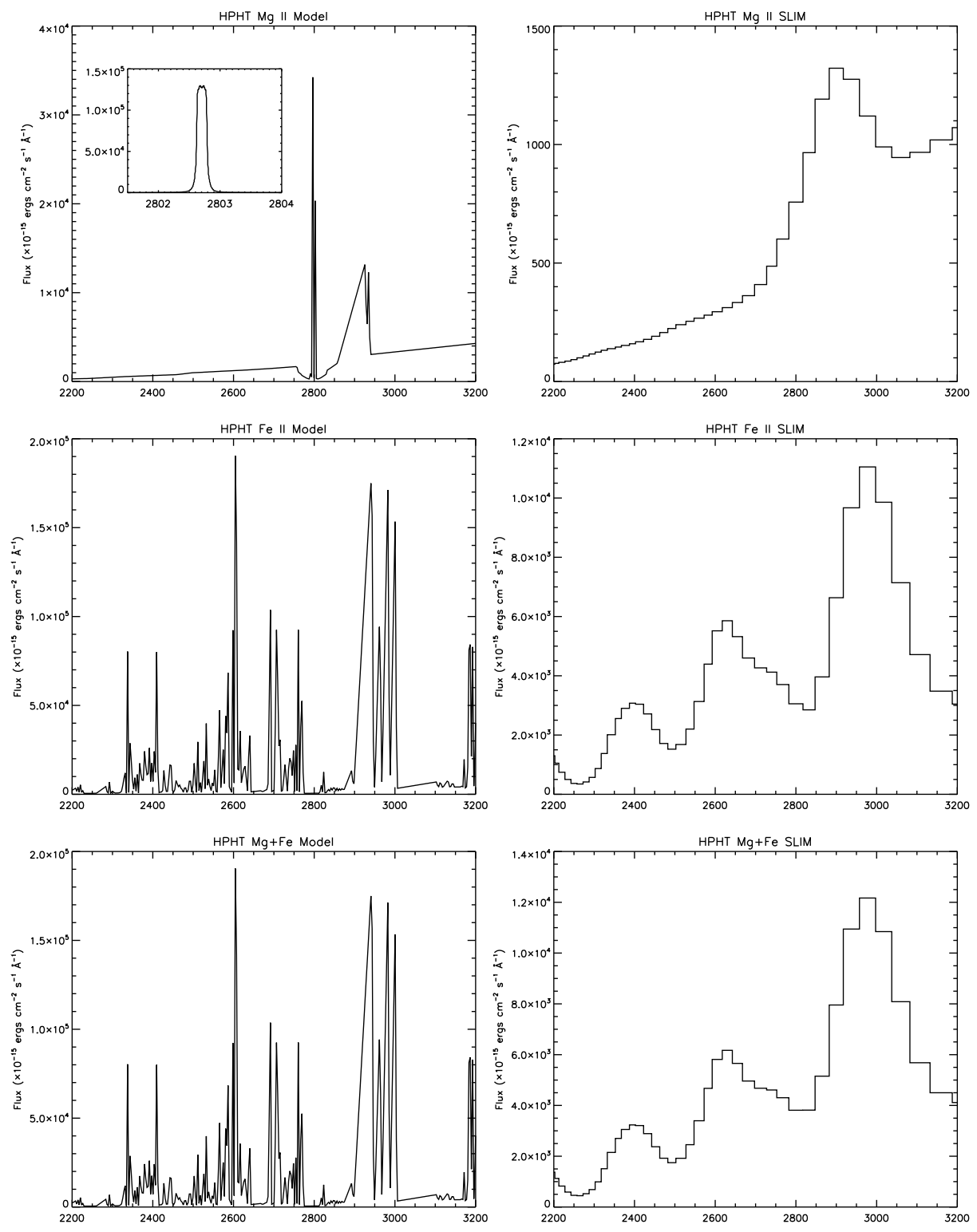

FIG. 10.- Mg II and Fe II Model results for HPHT atmosphere, degraded to ACS PR200L resolution using SLIM. 
Walkowicz et al.
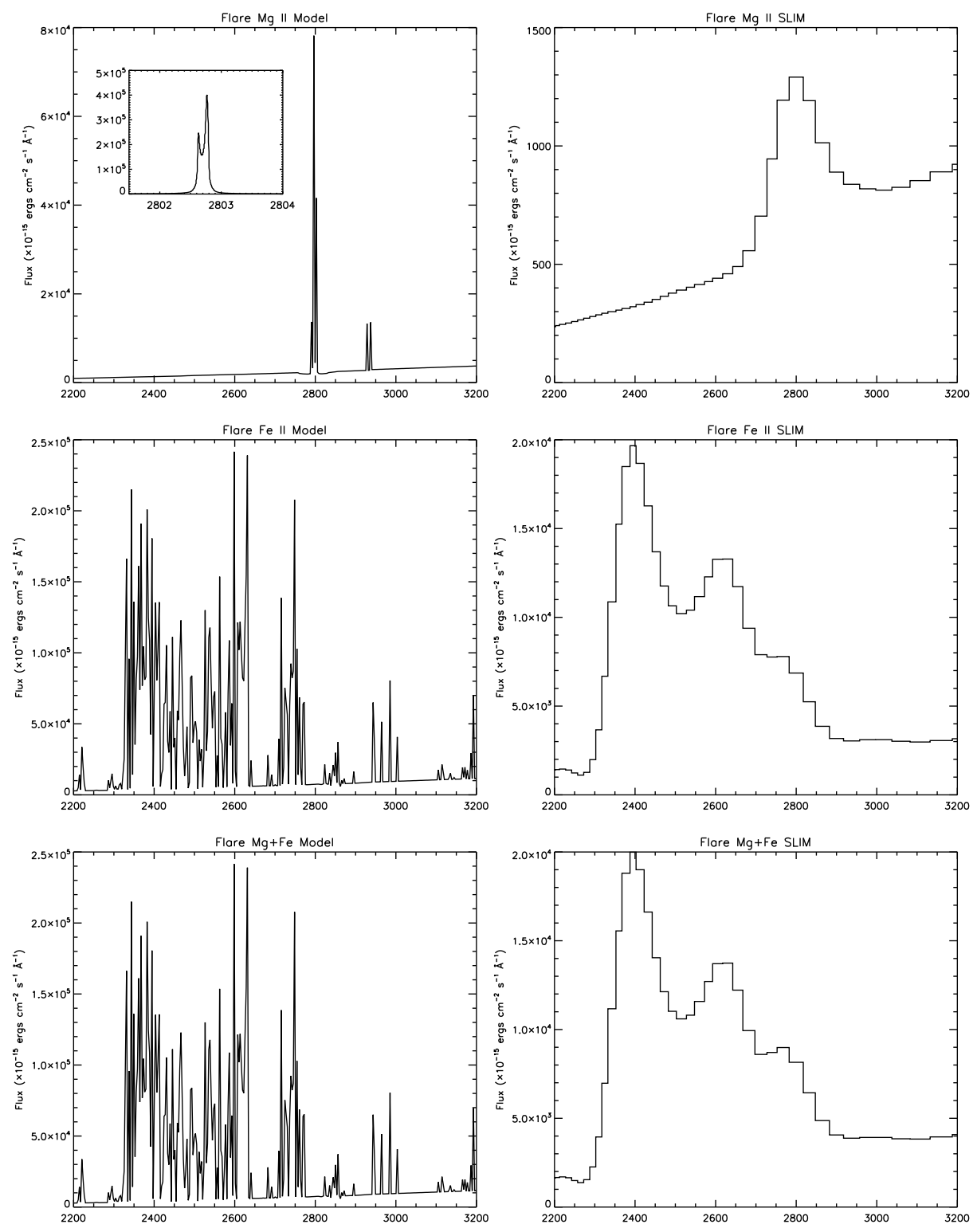

FIG. 11.- Mg II and Fe II Model results for Flare atmosphere, degraded to ACS PR200L resolution using SLIM. 


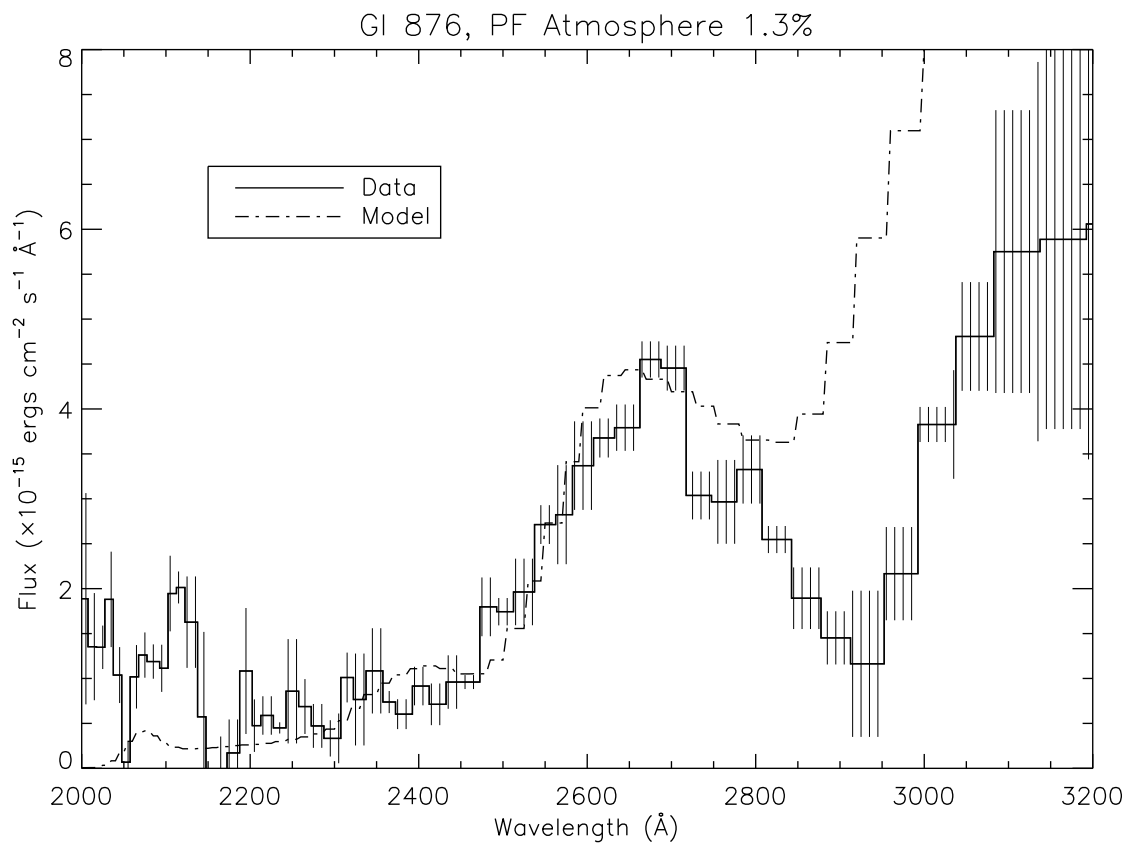

FIG. 12.- Mg II and Fe II Model results for Gl 876. The spectrum is best fit with the "PF" atmosphere at a fill factor of $1.3 \%$. These results show a substantial near-UV flux for even low activity M dwarfs, and are discussed in light of recent habitability models in Section 3.3.1.
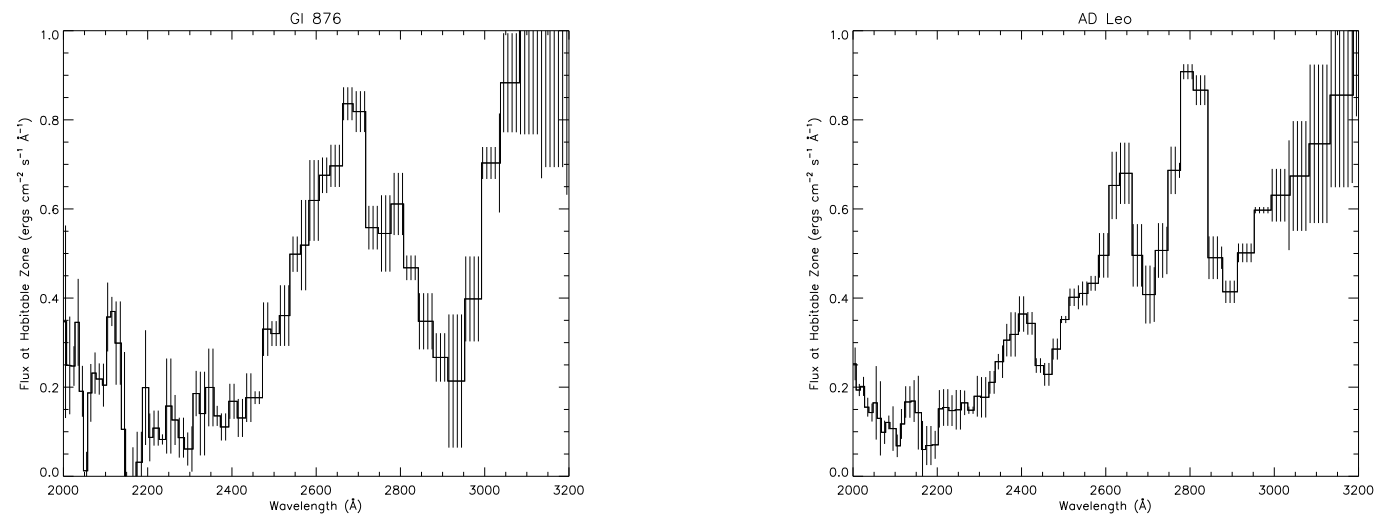

Fig. 13. - Near-UV flux at the habitable zone for Gl 876 and AD Leo. The stellar fluxes are scaled to habitable zone distances given in Segura et al. (2005). Although Gl 876 has substantially less activity than AD Leo, its near-UV flux combined with the habitable zone's proximity conspires to make the near-UV top-of-atmosphere flux comparable to AD Leo. 


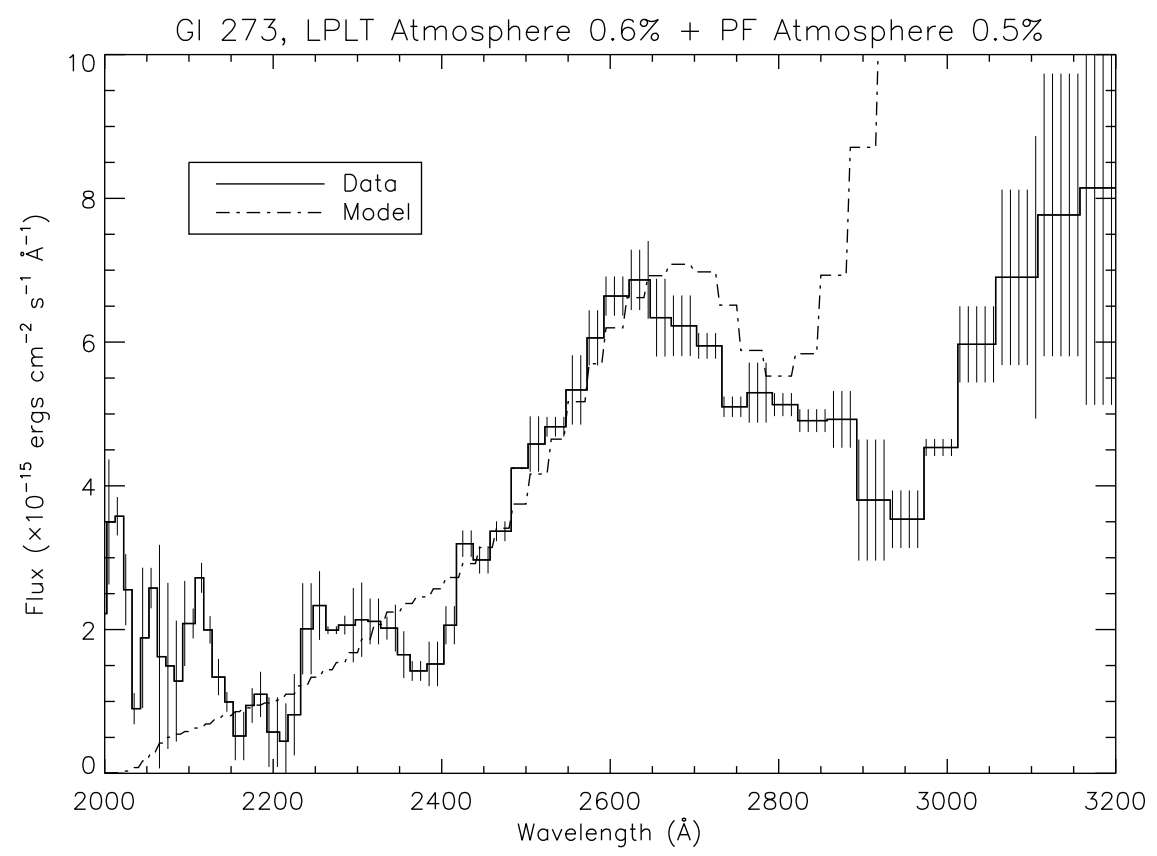

FIG. 14. - Mg II and Fe II Model results for Gl 273. The spectrum is best fit by a combination of LPLT and PF atmospheres in approximately equal portion $(0.6 \%$ and $0.5 \%)$.

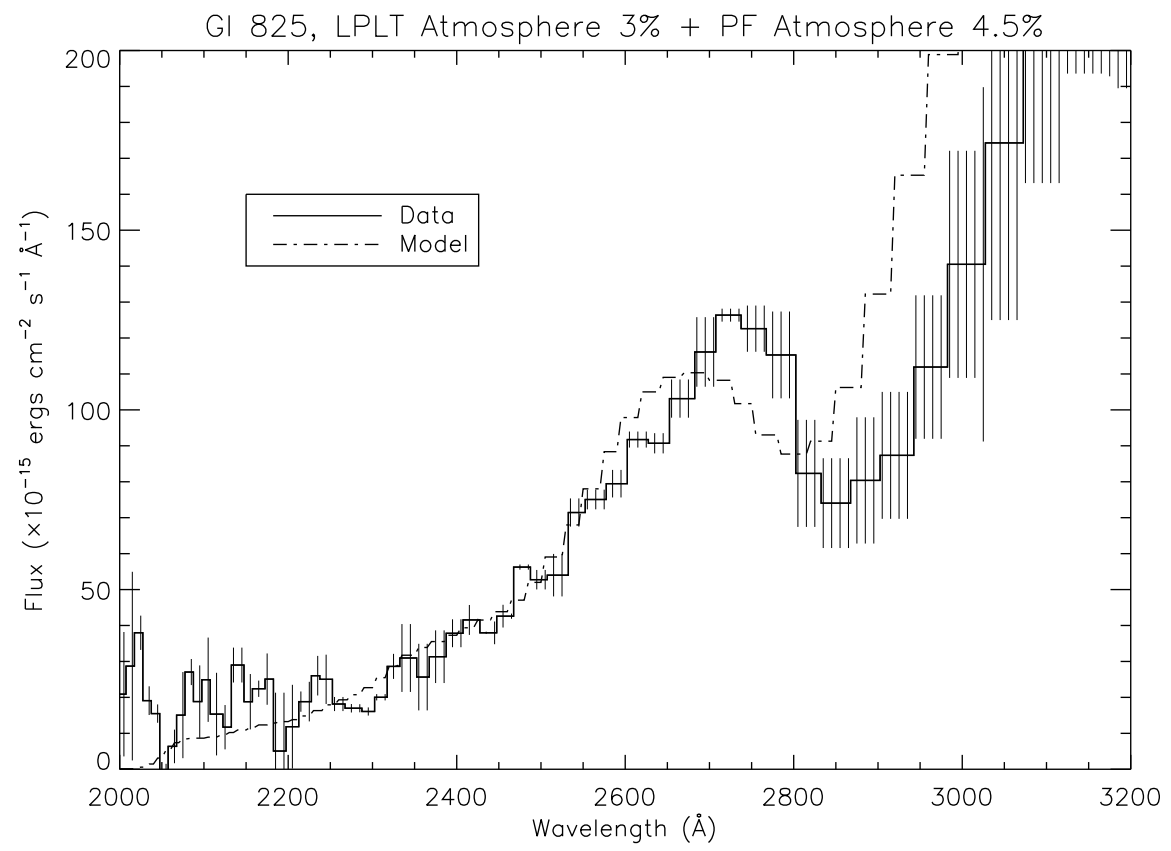

Fig. 15.- Mg II and Fe II Model results for Gl 825. The LPLT and PF atmosphere results at fill factors of $3 \%$ and $4.5 \%$ provide a good fit across the majority of the spectrum, though the peak in flux around $2750 \AA$ is higher than predicted by the models. 


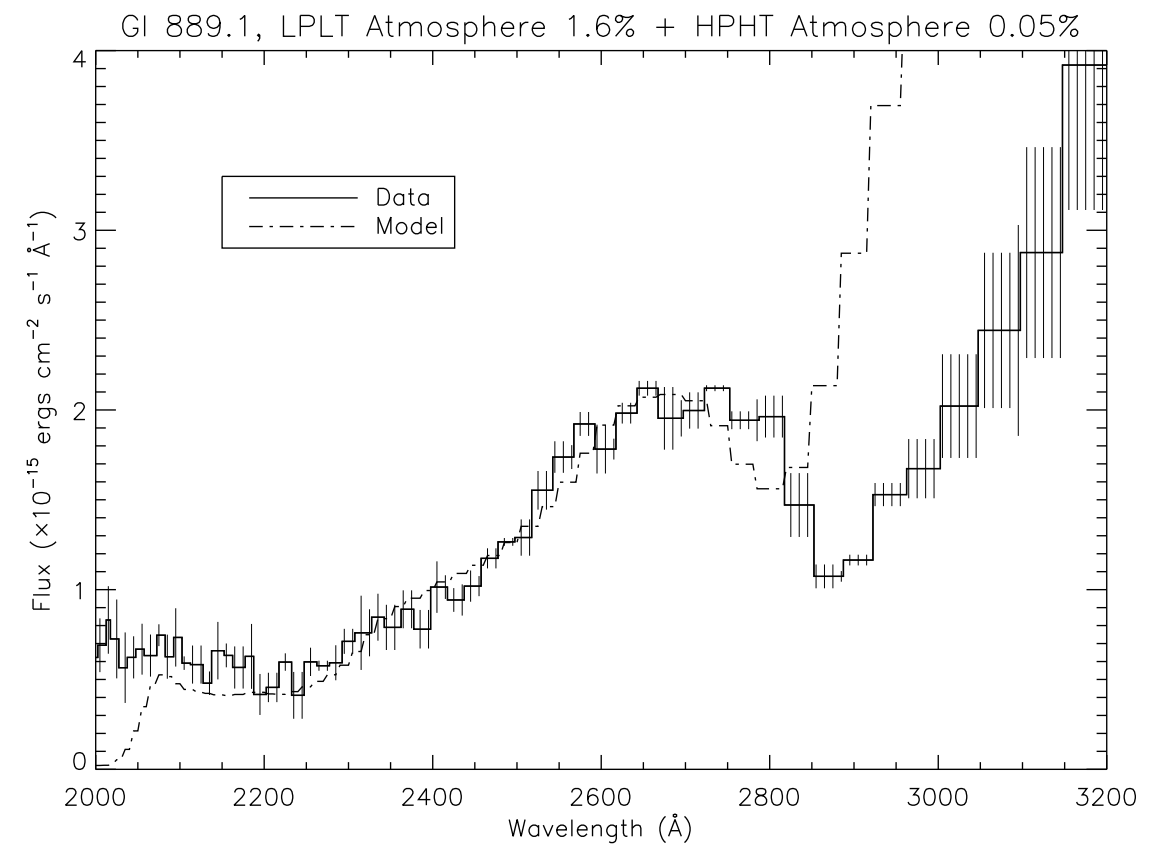

FIG. 16. - Mg II and Fe II Model results for Gl 889.1. The overall shape of the spectrum is reminiscent of Gl 825 and is well fit by the LPLT atmosphere (1.6\%), but the hotter HPHT component must also be included $(0.05 \%$ to match the short wavelength flux from 2000 to $2200 \AA$.
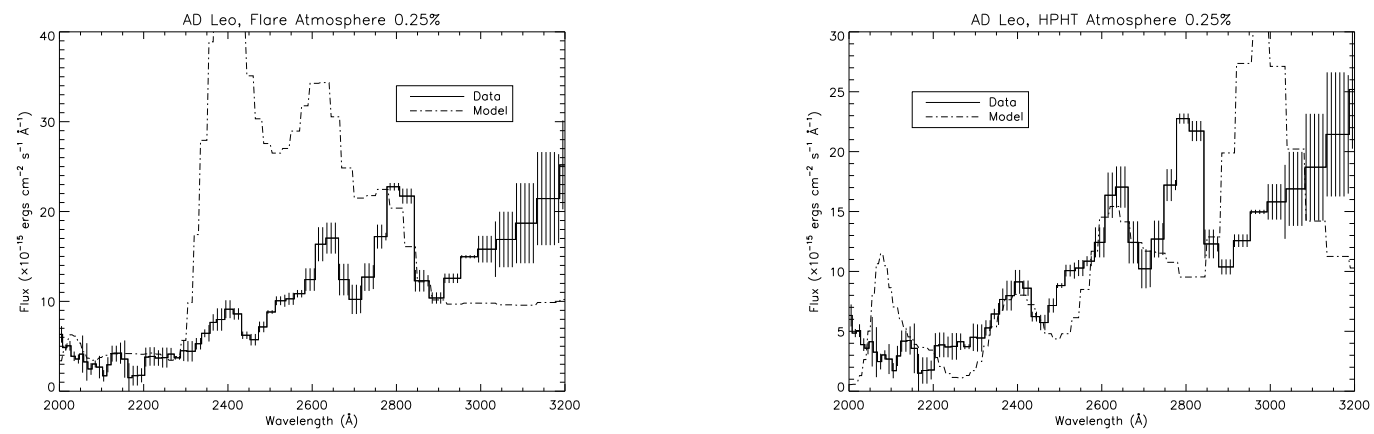

FIG. 17.- Mg II and Fe II Model results overplotted with AD Leo data- left: Flare atmosphere, right: HPHT atmosphere. Our hottest "Flare" model, with a fill factor of $0.25 \%$, roughly matches the flux around $2800 \AA$ and at wavelengths shorter than $2200 \AA$, but the Fe II emission at $\sim 2400 \AA$ and $\sim 2600 \AA$ is vastly overestimated. The HPHT atmosphere (also at a fill factor of $0.25 \%$ ) produces a much better match to these two Fe II features, but unfortunately does not account for any of the observed Mg II flux. 